IASSNS-HEP 92/11

MIT-CTP-2072

February 1992

\title{
THE PLUMBING OF MINIMAL AREA SURFACES
}

\author{
Michael WolF ${ }^{\star}$ \\ Department of Mathematics \\ Rice University \\ Houston, Texas 77251, U.S.A.
}

\author{
BARTON ZWIEBACH $^{\dagger}$ \\ School of Natural Sciences \\ Institute for Advanced Study \\ Olden Lane \\ Princeton, New Jersey 08540, U.S.A.
}

\begin{abstract}

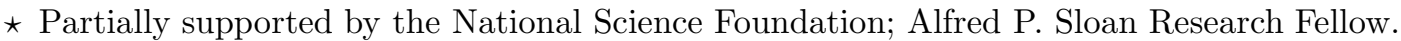

$\dagger$ Permanent address: Center for Theoretical Physics, MIT, Cambridge, Mass. 02139.

Supported in part D.O.E. contract DE-AC02-76ER03069 and NSF grant PHY91-06210.
\end{abstract}


We study the metric of minimal area on a punctured Riemann surface under the condition that all nontrivial homotopy closed curves be longer than or equal to $2 \pi$. By constructing deformations of admissible metrics we establish necessary conditions on minimal area metrics and a partial converse to Beurling's criterion for extremal metrics. We explicitly construct new minimal area metrics that do not arise from quadratic differentials. Under the physically motivated assumption of existence of the minimal area metrics, we show there exist neighborhoods of the punctures isometric to a flat semiinfinite cylinder of circumference $2 \pi$, allowing the definition of canonical complex coordinates around the punctures. The plumbing of surfaces with minimal area metrics is shown to induce a metric of minimal area on the resulting surface. This implies that minimal area string diagrams define a consistent quantum closed string field theory. 


\section{Introduction and Summary}

The motivation for the present work is a minimal area problem for Riemann surfaces. Given a Riemann surface $\mathcal{R}$ the problem asks for the conformal metric of least possible area under the condition that all homotopically nontrivial closed curves on the surface be longer than or equal to a fixed length, conventionally taken to be $2 \pi$ [Zw1]. The surface $\mathcal{R}$ is a surface of genus $g \geq 0$ with $n \geq 0$ marked points, or punctures, and the homotopy type of the curves is relative to the punctures. The cases of Riemann spheres with one or no punctures must be excluded since these surfaces have no nontrivial closed curves.

This generalized minimal area problem can be viewed as an extremal length problem. As is well known, extremal length problems in Riemann surfaces begin with the specification of a family $F$ of curves in a surface [Ah,Beu,Ga]. The extremal length, which is a conformal invariant, depends on the choice of the family $F$. The present problem, as will be explained in $\S 2$, is equivalent to an extremal length problem where $F$ is the set of all nontrivial closed curves in the surface. As such, the extremal length will be a function of the moduli of the surface only. This problem can also be viewed as a generalization of the minimal area problems studied earlier by Jenkins and Strebel [Je,St] where the length conditions apply to curves homotopic to a finite set of curves, called an admissible set, containing nonintersecting, non-homotopic, and nontrivial simple closed Jordan curves. Different length conditions may apply for the different homotopy types. In the present problem we impose the same length condition on all nontrivial closed curves. ${ }^{\star}$ In other words the unique length condition applies to curves homotopic to a curve in the infinite set containing a representative from every homotopy class. The curves in this set intersect and do not make an admissible set.

The solution of the generalized minimal area problem is known for all Riemann spheres $(g=0 ; n \geq 2)$, namely, all surfaces in the Riemann moduli spaces $\mathcal{M}_{g=0, n}$,

\footnotetext{
$\star$ It is actually sufficient to consider all nontrivial simple Jordan closed curves [Zw3], since nontrivial curves with self intersections will satisfy the length condition once all simple closed curves do.
} 
and for a subset of every $\mathcal{M}_{g, n}$ for $g \geq 1$ [Zw1]. The metrics arise from JenkinsStrebel (JS) quadratic differentials (quadratic differentials with closed horizontal trajectories) with second order poles at the punctures and with characteristic ring domains that include a punctured disc around each puncture, and a variable number of internal annuli. The horizontal trajectories, all of which are of length $2 \pi$, completely foliate the surface, and are geodesics that saturate the length conditions. We do not presently know the minimal area metrics for some subsets of every $\mathcal{M}_{g, n}$ (with the exception of $g=1, n=0$ ). There was some evidence that the minimal area metrics would not arise from JS-quadratic differentials [Zw2], and in $\S 6$ we describe such an example.

The relevance of the generalized minimal area problem for physics arises in the context of developing a second quantized field theory of closed strings [SaZw, KKS, KS]. The existence of such a field theory demands, roughly speaking, that we find for every $\mathcal{M}_{g, n}$ (except $g=0 ; n=0,1$ ), a subset $\mathcal{V}_{g, n}$, and for each surface in this subset we must specify a local coordinate $z_{i}$ around each of the punctures (this coordinate is specified only up to a phase). The subsets $\mathcal{V}$ define the vertices of the field theory. The fundamental constraint they must satisfy is the following: if we glue together the surfaces in the subsets $\mathcal{V}$, via the plumbing relations $z_{i} w_{i}=t$ with $|t| \leq 1$, and obeying the combinatorial rules of Feynman diagrams, we must generate precisely the complete moduli spaces $\mathcal{M}_{g, n}$ [SoZw, Zw4]. The metrics solving the generalized minimal area problem are expected to determine the subsets $\mathcal{V}$ and tell us how to put coordinates around the punctures of the corresponding surfaces [Zw2]. Basically, the minimal area metric is expected to be isometric to a flat semiinfinite cylinder around each puncture, this flatness requirement allowing us to define a canonical coordinate. Since area is additive, the plumbing of minimal area metrics is expected to induce in the plumbed surface a minimal area metric. This is true if minimal area metrics satisfy an amputation property [Zw2]: amputation of the semiinfinite cylinder associated to a puncture

$\dagger$ The specific metrics conjectured in [Zw2] to give such an example are now known not to be of minimal area. 
along a geodesic must induce on the truncated surface a metric of minimal area. If plumbed surfaces acquire minimal area metrics, the uniqueness of minimal area metrics [St,Zw1] will imply that Feynman rules will not generate any surface more than once. The $\mathcal{V}$ 's are defined to be the surfaces that are not generated by the sewing procedure. There is a more explicit description of $\mathcal{V}$ in terms of the heights of foliations in the metric of minimal area $[\mathrm{Zw} 5]$.

The relevance of the generalized minimal area problem for mathematics arises from the possibility of parametrizing the moduli spaces of surfaces, and in particular, the compactification divisors of moduli space in a canonical and geometrical way. Now, the Deligne-Mumford compactification $\overline{\mathcal{M}}_{g}$ of the moduli space of surfaces $\mathcal{M}_{g}(g>1)$ consists of a union of the moduli space of surfaces with a compactifying set of noded Riemann surfaces, i.e. connected complex spaces where points have neighborhoods complex isomorphic to either $\{|z|<\epsilon\}$ (regular points) or $\{z w=0 ;|z|<\epsilon,|w|<\epsilon\}$ (nodes), and for which each component of the complement of the nodes has negative Euler characteristic (note that the complement of the nodes is a collection of surfaces with paired punctures). The topology of the compactification and a parametrization of neighborhoods of points representing noded surfaces in $\overline{\mathcal{M}}_{g}$ are given by the process of conformal plumbing or "opening of a node" (see [Mas],[EM],[Ber]); we describe the process for a neighborhood in $\overline{\mathcal{M}}_{g}$ of a surface $\mathcal{R}_{0}$ with a single node, leaving the general case to the reader. To begin, for $|t|<\epsilon$ we remove a small neighborhood $\mathcal{U}=\{|z|<|t|,|w|<|t|\}$ of the node in the noded Riemann surface $\mathcal{R}_{0}$ and form the identification space $\mathcal{R}_{t}=\left(\mathcal{R}_{0}-\mathcal{U}\right) /<z w=t>$. We observe that $\mathcal{R}_{t}$ is a smooth compact Riemann surface. The topology of $\overline{\mathcal{M}}_{g}$ is then defined so that points in $\overline{\mathcal{M}}_{g}$ which are near $\mathcal{R}_{0}$ are either those noded surfaces which are quasiconformally close to $\mathcal{R}_{0}$, or those smooth surfaces which are the result of opening the node (with small opening parameter $t \neq 0$ ) of noded surfaces quasiconformally near $\mathcal{R}_{0}$.

Now, these coordinates we have described are not canonically defined, involving a choice of a neighborhood $\mathcal{U}$. However, one of the goals in this paper is to show that, for extremal metrics that are complete and sufficiently smooth in a sufficiently 
large neighborhood of the puncture, we can make the above process of opening the node somewhat more canonical: there will be a maximal neighborhood of the puncture which is flat and foliated by geodesics of length $2 \pi$ homotopic to the punctures. Taking this neighborhood to be a disk of radius one, where the geodesics are the circles of constant radius, we define $\mathcal{R}_{t}$ in a manner that depends only on the choice of that particular point of the boundary of the disk that should represent $z=1$. (In this choice, we must have an ambiguity as to the $z$-argument of that point because of the topology of $\mathcal{T}^{*} \overline{\mathcal{M}}_{g}$, see [W, Remark 4.1, p. 525]). Moreover, our results imply that the minimal area metric on the smooth surface $\mathcal{R}_{t}$, for $|t|<\exp (-\pi)$, is precisely the metric induced on $\mathcal{R}_{t}$ by the identification process. Thus minimal area metrics are consistent with the conformal process of opening a node, and dually, with degeneration in $\overline{\mathcal{M}}_{g}$ (pinching off a curve to obtain a noded surface). In physics this translates into manifest off-shell factorization of string amplitudes, an essential property of covariant field theory.

Since minimal area metrics will not arise always from quadratic differentials, we expect to find a natural and interesting generalization of quadratic differentials. We remark that there is presently no known assignment of quadratic differentials to all surfaces consistent with degeneration. Moreover, the minimal area problem applies to surfaces without punctures, where quadratic differentials do not yield decompositions of the corresponding moduli spaces (see [Ha]). The overall goal is for minimal area metrics to give a decomposition of all moduli spaces. Each moduli space would be broken into the pieces generated by the different patterns of foliations, these pieces roughly correspond to the various Feynman graphs of the string field theory. More explicit knowledge of the minimal area metrics may be necessary to decide whether the decomposition is actually a cell decomposition. This is the case for the moduli spaces $\overline{\mathcal{M}}_{0, n}[\mathrm{Zw} 1]$.

The goal of the present work is to provide a mathematical framework for the generalized minimal area problem. We establish a useful partial converse to a criterion of Beurling for extremal metrics. This amounts to necessary conditions for extremal metrics. In this paper we do not prove the existence of the minimal area 
metric on an arbitrary Riemann surface. The above results allow us to give, assuming the existence of a complete minimal area metric smooth in some neighborhood of each puncture, a proof of the requirements of flatness and amputation. This amounts to establishing (modulo existence) that the minimal area metrics define a quantum closed string field theory.

We have been able to obtain some minimal area metrics solving our problem that do not arise from quadratic differentials. The new property is that foliations by geodesics of length $2 \pi$ that cover the surface must intersect over regions of nonzero measure. Since these metrics are quite novel, and provide further evidence of existence of the minimal area metric for arbitrary Riemann surfaces, we will present them in $\S 6$.

The contents of this paper are organized as follows. In $\S 2$ we discuss preliminaries and notation. We explain the relation with extremal length and the notion of saturating geodesics. It is shown that for a complete minimal area metric, smooth and nonvanishing in some neighborhoods of the puctures, we have a foliation by saturating geodesics around the punctures. In $\S 3$ we prove a lemma that amounts to a (partial) local converse to Beurling's criterion [Beu]. While Beurling's criterion is a sufficient condition for a metric to be of minimal area, our converse gives necessary conditions. Roughly speaking, the necessary conditions apply if a minimal area metric $\rho_{0}$ is smooth on a compact domain which is foliated by saturating geodesics $\gamma_{i}$. Then, for any smooth $h$ in this domain such that $\int_{\gamma_{i}} h|d z| \geq 0$, one must have $\int h \rho_{0} \mathrm{dxdy} \geq 0$. Here the saturating geodesics may belong to a finite number of homotopy classes of curves. Using this criterion we show flatness near the punctures in $\S 4$. The proof of amputation is given in $\S 5$, where we also review the proof that sewing of minimal area metrics yield minimal area metrics [ $\left.\mathrm{Zw}_{\mathrm{w} 2}\right]$. The new minimal area metrics which do not correspond to quadratic differentials are given in $\S 6$.

In an interesting paper K. Ranganathan [Ra] has independently found a criterion for minimal area metrics in regions foliated by a single homotopy class of 
geodesics. His result, as we will see, also establishes flatness around the punctures. Since the lemma presented in $\S 3$ since does not assume a single foliation, it may be useful to determine if regions of the surface with multiple foliations are also flat.

\section{Preliminary Notions}

We will be concerned throughout with conformal metrics on Riemann surfaces. A (conformal) metric $\rho(z)|d z|$ must be invariantly defined, i.e. $\rho\left(z_{1}\right)\left|d z_{1}\right|$ $=\rho\left(z_{2}\right)\left|d z_{2}\right|$, where $z_{1}$ and $z_{2}$ are local parameters. The conformal factor $\rho$ must be measurable and non-negative everywhere. The length of a curve $\gamma$, denoted as $l_{\rho}(\gamma)$ is given by $\int_{\gamma} \rho|d z|$, and the area of the metric $\rho$ is given by $\iint \rho^{2} d x d y$. Both length and area are invariantly defined. The minimal area problem we are considering is the following [Zw1]:

Generalized Minimal Area Problem. Given a genus $g$ Riemann surface, with $n \geq 0$ punctures $(n \geq 2$ for $g=0$ ) find the metric of minimal area under the condition that the length of any nontrivial homotopy closed curve be greater than or equal to $2 \pi$.

In the introduction we have reviewed some properties of the minimal area metric. Here we shall concentrate on other aspects of this problem, of direct relevance for our present work.

Whenever there are punctures the naive definition of area is not adequate for the above problem. One must use the "reduced area" defined as [Zw1] (see also [St] $\S 3.2$ )

$$
\mathcal{A}(\rho)=\lim _{r \rightarrow 0}\left(\iint_{\mathcal{R}(r)} \rho^{2} d x d y+2 \pi n \ln r\right),
$$

where $n$ is the number of punctures and $\mathcal{R}(r)$ denotes the Riemann surface obtained by excising the disks $\left|z_{j}\right| \leq r$ around the punctures. The $z_{j}$ 's are arbitrary (but fixed) local coordinates vanishing at the punctures. Under a change of local 
coordinates the reduced area changes by a metric independent constant, and therefore the notion of a metric of minimal reduced area is independent of the choice of local coordinates.

A metric $\rho$ is called admissible (for the minimal area problem) if all nontrivial curves satisfy the length conditions. A curve satisfying the length conditions will be called a "good" curve; a curve that violates the length conditions will be called a "bad" curve. Let us emphasize that we will always be dealing with simple closed curves, i.e. curves without self-intersections.

Given two admissible metrics $\rho_{0}$ and $\rho_{1}$ the metric $\rho_{t}=(1-t) \rho_{0}+t \rho_{1}$ is admissible for all $t \in[0,1]$. The reduced area can be shown to be a strictly convex functional: $\mathcal{A}\left(\rho_{t}\right)<(1-t) \mathcal{A}\left(\rho_{0}\right)+t \mathcal{A}\left(\rho_{1}\right)$ for $t \in(0,1)$. This implies that the metric of minimal reduced area is unique $[\mathrm{Zw} 1]^{\star}$

Let us now explain the relation to extremal length. Consider, for simplicity, a genus $g$ surface $(g \geq 1)$ with no punctures (so that we can use area rather than reduced area). Given a family $F$ of curves on the surface, the extremal length $\Lambda(F)$ is a conformal invariant defined by

$$
\Lambda(F)=\sup _{\rho} \frac{L(\rho)^{2}}{A(\rho)}
$$

where the supremum is taken over all possible metrics, $L(\rho) \equiv \inf _{\gamma \in F} l_{\rho}(\gamma)$, and $A(\rho)$ is the area. Since the extremal length does not change under $\rho \rightarrow c \rho$, with $c$ an arbitrary positive constant, it is possible for every $\rho$ to demand $L(\rho)=2 \pi$, namely that all curves in $F$ be longer or equal to $2 \pi$. Then, it follows from (2.2) that we must try to make the area $A$ as small as possible. Thus the metric of least area under the condition that all curves in $F$ be longer than or equal to $2 \pi$ (if it exists) will give us the value of $\Lambda(F)$. Our minimal area problem simply corresponds to the case when $F$ is the set of all nontrivial curves on the surface.

$\star$ This property is well known for the ordinary definition of area. See, for example, K. Strebel $[\mathrm{St}]$. 
Remark. The quantity $f=(2 \pi)^{2} / A(\rho)$ for the minimal area metric is expected to define an interesting function $f: \overline{\mathcal{M}}_{g, 0} \rightarrow \mathbf{R}$. It is a function on moduli space since it is unique and requires no choice of curves. At the compactification divisor it is expected to go to zero since the area must diverge. Example: it is not hard to show that for $g=1$, in terms of the modular parameter $\tau$ in the usual fundamental domain $-1 / 2 \leq \tau<1 / 2,|\tau| \geq 1$ and $\operatorname{Im}(\tau)>0$; the function $f$ is simply $f=1 / \operatorname{Im}(\tau)$. The torus with least minimal area has $\tau=\exp (i \pi / 3)$.

An important notion for us will be that of saturating geodesics. These are the generalization, for our problem, of the closed horizontal trajectories of JenkinsStrebel quadratic differentials. They are simply the geodesics of length $2 \pi$, namely the geodesics that saturate the length conditions of the minimal area problem. We now show that under some conditions the saturating geodesics give rise to a foliation.

Lemma 2.1. Consider a punctured Riemann surface $\mathcal{R}$ with a complete minimal area metric $\rho_{0}$, smooth and nonvanishing in some neighborhood $N_{i}$ of each puncture $p_{i}$. Then there is a neighborhood $V_{i}$ around each puncture $p_{i}$ which is foliated by saturating geodesics homotopic to the puncture.

Proof. Consider a puncture $p$ surrounded by a neighborhood $N$ in which $\rho_{0}$ is smooth and non-vanishing. Then let $\gamma_{0} \subset N$ be an embedded (simple) closed curve homotopic to the puncture. Let $V$ be the neighborhood of the puncture consisting of points which are at distance greater than $3 \pi$ from $\gamma_{0}$. Completeness of the metric guarantees the existence of the neighborhood $V$ : if no such neighborhood could be found it would mean that every neighborhood of the puncture would contain a point whose distance to a fixed point $x_{0}$ in the curve $\gamma_{0}$ is smaller than $3 \pi+l\left(\gamma_{0}\right) / 2$, and then we would have a sequence of points converging to the puncture whose distance to a fixed point is bounded, in contradiction with the completeness of $\rho_{0}$.

Then we claim that if $C$ is a nontrivial closed curve which intersects $V$ but which is not homotopic to $\gamma_{0}$, then $l_{\rho_{0}}(C)>3 \pi$. To see this, notice that $C$ must intersect $\gamma_{0}$, and the distance from $\gamma_{0}$ to any point in $V$ is at least $3 \pi$. Thus if 
there is a saturating geodesic intersecting $V$ it must be homotopic to the puncture.

We claim that there is a smooth curve through $q$ homotopic to $p$ of length $2 \pi$. We first observe that there must be a curve passing through $q$ of length less than or equal to $2 \pi+\epsilon$, for every $\epsilon>0$ [Zw2]; if this were not true, then there is some $\epsilon$ such that all nontrivial closed curves through $q$ are longer than $2 \pi+\epsilon$. Consider then an $\epsilon / 3$ neighborhood of $q$, and set the metric equal to zero throughout this neighborhood. We claim the new metric is admissible. If any curve becomes shorter than $2 \pi$, it is because its portion lying outside the neighborhood is smaller than $2 \pi$, but then the nontrivial open subcurve lying outside the neighborhood could be made into a nontrivial closed curve by joining its endpoints with $q$. In this way we would get a curve of length smaller than $2 \pi+2 \epsilon / 3$ in contradiction with the assumption that all nontrivial closed curves through $q$ had to be longer than $2 \pi+\epsilon$.

We consider now a family of curves $\gamma_{n}$ passing through $q$ of lengths less than $2 \pi+\frac{1}{n}$. All of these curves are homotopic to the puncture $p$, since they all have lengths less than $3 \pi$. Now, extend $\left.\rho_{0}\right|_{N}$ to a smooth complete admissible metric $\rho_{0}$ on $\mathcal{R}$ and then lift this metric to a metric $\widetilde{\rho}_{0}$ on the universal cover $\widetilde{\mathcal{R}}$. Then, by the Hopf-Rinow theorem, we find a minimal geodesic $\widetilde{\gamma}$ between the endpoints of a lift $\widetilde{\gamma}_{n}$ of $\gamma_{n}$. Of course, this geodesic $\widetilde{\gamma}$ must have length less than $2 \pi+\frac{1}{n}$, for all $n$, since it is minimizing. Moreover, since $\widetilde{\gamma}$ will project to a curve homotopic to the puncture and $\rho_{0}$ is admissible, the curve $\gamma$ must have length at least $2 \pi$. We conclude that this curve has length exactly $2 \pi$, and thus, by the arguments above, $\gamma$ lives in $N$, and is a $\rho_{0}$-geodesic. Finally, $\gamma$ is smooth at $q$, since otherwise we could cut any corner of $\gamma$ at $q$ and obtain a curve of length less than $2 \pi$, thus showing that $\rho_{0}$ is not admissible.

If there is more than one saturating geodesic through $q$ they would have to intersect at some finite angle, since in a smooth metric two geodesics going through the same point and having the same first derivative must coincide completely. If two saturating geodesics intersect they would have to do so at least at two points. Consider then two homotopic segments determined by the intersection 
points. These must have equal lengths, and then, a cut and paste argument shows that the length of the curves could be reduced at the intersection points. Thus we have established the uniqueness of the saturating geodesic going through $q$ and the fact that homotopic geodesics cannot intersect. This implies that the neighborhood $V$ will be foliated completely by saturating geodesics homotopic to the puncture.

\section{The Deformation of an Admissible Metric}

In this section we will establish a lemma that describes deformations that can be made to admissible conformal metrics (in a region covered by a finite number of foliations) so that the deformed metric will remain admissible. This lemma and its corollary will be used in $\S 4$ to show that if a minimal area metric were not flat in a neighborhood of each puncture, then we could flatten it slightly and reduce its area, contradicting the presumed minimality of the metric.

Lemma 3.1. Let $\mathcal{R}$ be a Riemann surface and $\rho_{0}$ an admissible complete metric. Let $h$ be a smooth conformal metric (admitting both positive and non-positive values) and suppose that $h$ has compact support. Let $\Gamma$ denote the set of saturating geodesics passing through $\operatorname{supp}(h)$ and suppose that

(i) for every $\gamma_{0} \in \Gamma$, the set $\Gamma_{\left[\gamma_{0}\right]} \subset \Gamma$ of curves freely homotopic to $\gamma_{0}$ cover $\operatorname{supp}(h)$, i.e., $\operatorname{supp} h \subset \bigcup_{\gamma \in \Gamma_{\left[\gamma_{0}\right]}} \gamma$, for every $\left[\gamma_{0}\right]$ represented in $\Gamma$,

(ii) the original metric $\rho_{0}$ is smooth $\left(C^{\infty}\right)$ and non vanishing in a neighborhood of $\bigcup_{\gamma \in \Gamma} \gamma$

(iii) $\Gamma$ contains only a finite number of free homotopy classes of curves,

(iv) there is a $\delta_{0}$ so that if $\gamma$ is a nontrivial closed curve passing through $\operatorname{supp}(h)$ with $l_{\rho_{0}}(\gamma)<2 \pi+\delta_{0}$, then $\gamma$ is freely homotopic to a curve in $\Gamma$,

(v) $\int_{\gamma} h|d z| \geq 0$ for each $\gamma \in \Gamma$. 
Then there is an $\hat{\epsilon}$ and a constant $K$ independent of $\hat{\epsilon}$, such that for $\epsilon<\hat{\epsilon}$, the metric $\rho_{\epsilon}$ defined by

$$
\rho_{\epsilon}=\rho_{0}+\epsilon h+K \epsilon^{2} \chi
$$

is admissible. Here $\chi$ is a conformal metric which equals $\rho_{0}$ on a $2 \pi$ neighborhood of $\operatorname{supp}(h)$ and vanishes elsewhere.

Proof. Let $\widehat{\rho}_{\epsilon}$ denote the truncated $\rho_{\epsilon}$ metric,

$$
\widehat{\rho}_{\epsilon}=\rho_{0}+\epsilon h \text {. }
$$

Note that the metric $\widehat{\rho}_{\epsilon}$ is well defined only if $\epsilon$ is small enough (otherwise the metric could become negative). Since the support of $h$ is compact, over this support $\inf \left(\rho_{0}\right)$ exists and is different from zero (see (ii)), and sup $|h|$ exists and is different from zero or infinity. One then checks that for $\epsilon<\epsilon_{0}=\inf \left(\rho_{0}\right) / \sup |h|$, the metric $\rho_{\epsilon}$ is well defined.

We must check that all nontrivial closed curves on the surface are still longer than or equal to $2 \pi$ when the metric changes from $\rho_{0}$ to $\rho_{\epsilon}$, for $\epsilon<\epsilon_{0}$. We begin by narrowing the space of curves that must be checked. We now show that it is sufficient to consider only those curves $\gamma_{\epsilon}$ which satisfy the following four criteria: (a) $\gamma_{\epsilon}$ must intersect $\operatorname{supp}(h)$; otherwise its length can only increase when going from the $\rho_{0}$ to the $\rho_{\epsilon}$ metric.

(b) $\gamma_{\epsilon}$ must be contained in $\operatorname{supp}(\chi)$; if it is not, then, because of (a) the part of the curve outside $\operatorname{supp}(h)$ must have length of at least $2 \pi$. Since outside $\operatorname{supp}(h)$ we have $\rho_{\epsilon}>\rho_{0}$, the curve $\gamma_{\epsilon}$ has $\rho_{\epsilon}$-length at least $2 \pi$.

(c) The $\rho_{0}$ length of $\gamma_{\epsilon}$ must be less or equal to $4 \pi$, namely $l_{\rho_{0}}\left(\gamma_{\epsilon}\right) \leq 4 \pi$. Suppose it is not, then $l_{\rho_{0}}\left(\gamma_{\epsilon}\right)>4 \pi$. Consider now

$$
l_{\widehat{\rho}_{\epsilon}}\left(\gamma_{\epsilon}\right)=\int_{\gamma_{\epsilon}} \widehat{\rho}_{\epsilon}|d z|=\int_{\gamma_{\epsilon}} \frac{\widehat{\rho}_{\epsilon}}{\rho_{0}} \rho_{0}|d z|,
$$


for $\epsilon<\frac{1}{2} \epsilon_{0}$ one has

$$
\frac{\widehat{\rho}_{\epsilon}}{\rho_{0}}>\frac{1}{2}, \quad \text { so that } \quad l_{\widehat{\rho}_{\epsilon}}\left(\gamma_{\epsilon}\right)>\frac{1}{2} \int_{\gamma_{\epsilon}} \rho_{0}|d z|>\frac{1}{2} 4 \pi=2 \pi \text {, }
$$

which shows that the curve $\gamma_{\epsilon}$ will still be longer than $2 \pi$ in the modified metric. Thus indeed, we only need to check those curves whose $\rho_{0}$ length is shorter than $4 \pi$.

(d) The curve $\gamma_{\epsilon}$ is freely homotopic to an element of $\Gamma$. Note that only if $l_{\widehat{\rho}_{\epsilon}}\left(\gamma_{\epsilon}\right)<$ $2 \pi$ is there something to check, but in this case

$$
l_{\rho_{0}}\left(\gamma_{\epsilon}\right)+\epsilon \int_{\gamma_{\epsilon}} h|d z|<2 \pi
$$

and so,

$$
l_{\rho_{0}}\left(\gamma_{\epsilon}\right)<2 \pi+\epsilon \int_{\gamma_{\epsilon}}|h||d z|
$$

The last term above can be bounded

$$
\epsilon \int_{\gamma_{\epsilon}}|h||d z|=\epsilon \int_{\gamma_{\epsilon}}\left|\frac{h}{\rho_{0}}\right| \rho_{0}|d z| \leq \epsilon \frac{\sup |h|}{\inf \left(\rho_{0}\right)} 4 \pi \leq \delta_{0}
$$

for $\epsilon \leq \frac{\delta_{0}}{4 \pi} \epsilon_{0}$. We therefore have $l_{\rho_{0}}\left(\gamma_{\epsilon}\right)<2 \pi+\delta_{0}$ and the desired result is a consequence of hypothesis (iv). In summary we need only discuss curves contained in the support of $\chi$, and homotopic to elements of $\Gamma$.

We now begin our analysis of the relevant curves. Since $\gamma_{\epsilon}$ is freely homotopic to an element of $\Gamma$ and such elements cover $\operatorname{supp}(h)$, there is a curve $\gamma_{0} \in \Gamma$ which is homotopic to $\gamma_{\epsilon}$, intersecting $\gamma_{\epsilon}$ in at least two distinct points, say $p_{1}$ and $p_{2}$. To see this, observe that by (a) above, $\gamma_{\epsilon}$ must intersect the interior of $\operatorname{supp}(h)$, and so must intersect some $\gamma_{0} \in \Gamma$ which passes through the interior of $\operatorname{supp}(h)$. Then, if $\gamma_{0} \cap \gamma_{\epsilon}$ consists of only a single point, by replacing $\gamma_{0}$ with a nearby curve of the foliation near $\gamma_{0}$, we may assume that $\gamma_{0} \cap \gamma_{\epsilon}$ contains two points. 
Let $A_{0}$ and $A_{\epsilon}$ denote the arcs of $\gamma_{0}$ and $\gamma_{\epsilon}$, respectively, which pass through $p_{1}$ and $p_{2}$ and are homotopic rel $\left\{p_{1}, p_{2}\right\}$ (see Fig. 1). Our goal is to bound from below the $\widehat{\rho}_{\epsilon}$-length of $A_{\epsilon}$ in terms of the $\widehat{\rho}_{\epsilon}$-length of $A_{0}$, say $l_{\widehat{\rho}_{\epsilon}}\left(A_{\epsilon}\right)>l_{\widehat{\rho}_{\epsilon}}\left(A_{0}\right)-K_{3} \epsilon^{2}$, so that we can eventually bound the $\widehat{\rho}_{\epsilon}$-length of $\gamma_{\epsilon}$ in terms of the $\widehat{\rho}_{\epsilon}$-length of $\gamma_{0}$, which by hypothesis exceeds $2 \pi$.

To this end, set $\widehat{\rho}_{t}=\rho_{0}+t h$ and let $A_{t}$ denote the $\widehat{\rho}_{t}$-geodesic arc connecting $p_{1}$ and $p_{2}$; here $A_{0} \subset \gamma_{0}$. We wish to consider $l_{\widehat{\rho}_{t}}\left(A_{t}\right)$ as a function of $t$, and, in particular, we wish to differentiate this function in $t$. To begin this discussion, we first need to establish how $A_{t}$ varies with $t$. For this we recall the theorem of Eells and Lemaire [EL; $\S 4$ ] that for each pair of points $p_{1}$ and $p_{2}$ connected by a geodesic arc $A_{0}$, there is an $\epsilon_{0}$ (depending on the pair $\left(p_{1}, p_{2}\right)$ as well as $h$ and $\rho_{0}$ ) so that for $t<\epsilon_{0}$, we find a unique family of $\widehat{\rho}_{t}$-geodesic arcs $A_{t}$ connecting $p_{1}$ and $p_{2}$ as long as there are no non-trivial $\rho_{0}$-Jacobi fields along $A_{0}$ vanishing at the endpoints. Of course, there are no such Jacobi fields along $A_{0}$, because if there were, then there would be a non-trivial Jacobi field along $\gamma_{0}$, and we could use such a field to find a curve $\gamma_{0}^{*}$ of lower length than $\gamma_{0}$, contradicting the admissibility of $\rho_{0}$ (see, for example [Sp] Vol.4 Ch. 8 or [Hi] Ch. 10, Thm 11). Thus, we can find such an $\epsilon_{0}$ as described above; however, in order to argue for all extremal arcs $A_{\epsilon}$, we need an $\epsilon_{0}$ that does not depend on any particular $A_{0}$. To find such an $\epsilon_{0}$, we use the compactness of the space of endpoints $\left\{\left(p_{1}, p_{2}\right)\right\}$ as well as the smooth dependence of solutions of the Jacobi equation $J(V)=0$ upon the data. More precisely, let $N \subset \mathcal{R}$ denote the submanifold of $\mathcal{R}$ consisting of all points within $\operatorname{supp}(\chi)$. Here the completeness of $\rho_{0}$ implies that $N$ is a compact submanifold of $\mathcal{R}$. Form the compact product manifold $N \times N$ and consider within $N \times N$ the submanifold $X$ consisting of pairs of points $\left(p_{1}, p_{2}\right)$ with the property that $p_{1}$ and $p_{2}$ lie along a $\rho_{0}$-geodesic belonging to $\Gamma$, and that $d_{\rho_{0}}\left(p_{1}, p_{2}\right) \leq 2 \pi$. Now for each of the finite number of homotopy classes represented in $\Gamma$, there is a compact family of $\rho_{0}$-geodesics representing that homotopy class, and so we can conclude that $X$ is compact.

Finally, we want to form a compact space representing all the arcs of curves in 
$\Gamma$; to do this we need to account for there being two arcs in a curve in $\Gamma$ which have endpoints represented by $\left(p_{1}, p_{2}\right) \in X$. To this end, consider for each of the finitely many homotopy classes in $\Gamma$ a given fixed orientation, then we form the disjoint union $X_{0}=X \sqcup X$ so that the point $\left(p_{1}, p_{2}\right)$ in the first copy will correspond to the arc from $p_{1}$ to $p_{2}$ determined by the orientation of the underlying curve $\gamma \in \Gamma$ and $\left(p_{1}, p_{2}\right)$ in the second copy will correspond to the arc from $p_{1}$ to $p_{2}$ determined by the opposite orientation of the underlying curve $\gamma \in \Gamma$. The set $X_{0}$ is compact, as desired.

Now, for each curve $\gamma \in \Gamma$, we can consider the lift $\widehat{\gamma}$ to a unit speed periodic extension $\widehat{\gamma}: \mathbf{R} \rightarrow \mathcal{R}$ where $\widehat{\gamma}([0,2 \pi))$ covers the embedded curve $\gamma$ exactly once. Then, because there are no non-trivial Jacobi fields on $\gamma$, there is a $\delta(\gamma)$ so that there are no non-trivial solutions to the Jacobi equation with vanishing boundary values for $\widehat{\gamma}$ on the interval $[0, l]$ for $l<2 \pi+\delta(\gamma)$. Since $\Gamma$ is compact and the solutions to the Jacobi equation vary smoothly with the data, we conclude that there is a $\delta>0$ so that for all $\gamma \in \Gamma$, there are no non-trivial solutions to the Jacobi equation with vanishing boundary values for $\widehat{\gamma}$ on the interval $[0, l]$ for $l<2 \pi+\delta$.

From this uniform non-degeneracy of the Jacobi operator on boundary value problems with data from $X_{0}$ and the smoothness of $\rho_{0}$ near $\bigcup_{\gamma \in \Gamma} \gamma$, the proof of the Eells-Lemaire result implies that for every pair of points $x_{0}=\left(p_{1}, p_{2}\right) \in X_{0}$ there is a $T^{*}\left(x_{0}\right)$ and a neighborhood $\mathcal{N}$ of $x_{0}$ in $X_{0}$ so that for $|t|<T^{*}\left(x_{0}\right)$ and $x \in \mathcal{N}$, there is a unique and differentiable family of $\operatorname{arcs} A_{t}$ which are geodesic in the metric $\widehat{\rho}_{t}$ and have endpoints given by $x \in \mathcal{N} \subset X_{0}$. Using the compactness of $X_{0}$, we find a $T^{*}$ so that for $|t|<T^{*}$ and any pair of points $p_{1}, p_{2} \in \gamma \in \Gamma$, there is a unique and differentiable family of $\widehat{\rho}_{t}$-geodesic $\operatorname{arcs} A_{t}$ connecting $p_{1}$ and $p_{2}$. Also, the compactness of the family of arcs obtained for $|t| \leq T^{*} / 2$ together with the result of the last paragraph guarantees a $T_{0}$ with the same property as $T^{*}$ above but with the additional property that there do not exist any non-trivial $\widehat{\rho}_{t}$-Jacobi fields along any of the $\operatorname{arcs} A_{t}$ for $|t| \leq T_{0} / 2$.

We are interested in estimating the derivatives of the function $l_{\widehat{\rho}_{t}}\left(A_{t}\right)$, and it 
is here that the final property of the arcs $A_{t}$ described in the previous paragraph becomes important. We will need to know

$$
\left.\frac{\partial^{2}}{\partial t^{2}}\right|_{t=c} l_{\widehat{\rho}_{c}}\left(A_{t}\right)
$$

for all values $c$ with $|c|<\epsilon<T_{0} / 2$. It is convenient to introduce here the rectangular parameter space $(s, t)$ with $s \in[0,1]$ and $t \in(-\epsilon, \epsilon)$. We think of $A_{t}(s)$ of maps from the parameter space to the manifold, giving us, for constant $t$, the arc $A_{t}$. In addition, $A_{t}(0)=p_{1}$ and $A_{t}(1)=p_{2}$ for all $t \in(-\epsilon, \epsilon)$. The second variation formula for length provides that (3.2) can be bounded in terms of $\left\|W_{c}(s)\right\|_{\infty}$, where $W_{c}(s)=\left.\frac{\partial}{\partial t}\right|_{t=c} A_{t}(s)=A_{c *} \frac{\partial}{\partial t}$ is the tangent vector in the surface showing us how the geodesic segments move as we change $t$, and in terms of $\left\|\nabla_{\mathcal{T}_{c}}^{\widehat{\rho}_{c}} W_{c}\right\|_{\infty}$, where $\mathcal{T}_{c}=A_{c *} \frac{\partial}{\partial s}$ is the tangent vector to the geodesic arcs. We will now estimate those quantities.

To begin we consider a particular family $A_{t}$ with endpoints $\left(p_{1}, p_{2}\right) \in X_{0}$ which is geodesic in the metric $\widehat{\rho}_{t}$, and using a coordinate system $\left(x_{1}, x_{2}\right)$ in a neighborhood of the $\widehat{\rho}_{c}$-geodesic $A_{c}$, we compute the Christoffel symbols $\Gamma(t)=$ $\Gamma_{i j}^{k}(t)$ and find that for the conformal metric $\widehat{\rho}_{t}$, we may define a symbol $\Psi_{i j}^{k}(t)$ so that

$$
\Gamma_{i j}^{k}(t)=\Gamma_{i j}^{k}(c)+\Psi_{i j}^{k}(t)
$$

where $\Psi_{i j}^{k}(c)=0$. Moreover, since we can take $\widehat{\rho}_{t}$ non-vanishing in a neighborhood of $\bigcup_{\gamma \in \Gamma} \gamma$ which contains all of our $\operatorname{arcs} A_{t},|t|<T_{0} / 2$, and $h$ is smooth, we see that $\Psi_{i j}^{k}(t)$ is not only differentiable in $t$ but is also smooth in $\left(x_{1}, x_{2}\right)$, for $|t|<T_{0} / 2$. Then the $\widehat{\rho}_{t}$ geodesic arc $A_{t}$ satisfies

$$
\left(A_{t}\right)_{, s s}^{k}+\Gamma_{i j}^{k}(t)\left(A_{t}\right)_{, s}^{i}\left(A_{t}\right)_{, s}^{j}=0
$$

where each curve $A_{t}:[0,1] \rightarrow \mathcal{R}$ is parametrized proportionally to arc length. 
We rewrite (3.3) as

$$
\left(A_{t}\right)_{, s s}^{k}+\Gamma_{i j}^{k}(c)\left(A_{t}\right)_{, s}^{i}\left(A_{t}\right)_{, s}^{j}=\Psi_{i j}^{k}(t)\left(A_{t}\right)_{, s}^{i}\left(A_{t}\right)_{, s}^{j}
$$

Next we use $W_{c}(s)=\left.\frac{\partial}{\partial t}\right|_{t=c} A_{t}(s)$ and then differentiate (3.4) in the variable $t$ at $t=c$. It is straightforward (and classical) that the left hand side of (3.4) differentiates to give the Jacobi operator $\nabla_{\mathcal{T}} \nabla_{\mathcal{T}} W+R(W, \mathcal{T}) \mathcal{T}$ where we take the covariant derivatives and curvatures with respect to the metric at $t=c$, that is $\widehat{\rho}_{c}$. To evaluate the right hand side of (3.4), introduce $\widehat{\rho}_{c}$-Fermi coordinates along the arc $A_{c}$ so that $A_{c}(s)=\left(A_{c}^{1}, A_{c}^{2}\right)=\left(l_{\widehat{\rho}_{c}}\left(A_{c}\right) s, 0\right)$ and recall that $\Psi_{i j}^{k}(c)=0$. Then we find that

$$
\begin{aligned}
-\left.\frac{d}{d t}\right|_{t=c}\left[\Psi_{i j}^{k}(t)\left(A_{t}\right)_{, s}^{i}\left(A_{t}^{j}\right)_{, s}\right. & =-\left[\left.\frac{d}{d t}\right|_{t=c} \Psi_{11}^{k}(t)\right]\left[l_{\widehat{\rho}_{c}}\left(A_{c}\right)\right]^{2} \\
& \equiv \Psi^{k}\left(A_{c}(s)\right)\left[l_{\widehat{\rho}_{c}}\left(A_{c}\right)\right]^{2},
\end{aligned}
$$

where the last expression indicates a local function defined along the $\operatorname{arc} A_{c}(s)$. We are left with the vector equation

$$
J(W)=\nabla_{\mathcal{T}} \nabla_{\mathcal{T}} W+R(W, \mathcal{T}) \mathcal{T}=\vec{\Psi}\left(A_{c}(s)\right)\left[l_{\widehat{\rho}_{c}}\left(A_{c}\right)\right]^{2} .
$$

It is now convenient to introduce the vector field $T_{t}=\mathcal{T}_{t} / l_{\widehat{\rho}_{t}}\left(A_{t}\right)$, with unit normalization: $\left\langle T_{t}, T_{t}>^{1 / 2}=<\mathcal{T}_{t}, \mathcal{T}_{t}>^{1 / 2} /\left[l_{\widehat{\rho}_{c}}\left(A_{c}\right)\right]=1\right.$ (recall $\mathcal{T}_{t}=A_{t *} \frac{\partial}{\partial s}$, and $s \in[0,1])$. Therefore Eqn. (3.6) is rewritten as:

$$
\nabla_{T} \nabla_{T} W+R(W, T) T=\vec{\Psi}\left(A_{c}(s)\right)
$$

Now, by our choice of $|c|<T_{0} / 2$, we know that there are no Jacobi fields along $A_{c}$. Thus the general theory of ordinary differential equations ( $\mathrm{cf}[\mathrm{H}]$, Theorem XII.3.1) ensures a bound $K_{p_{1}, p_{2} ; c}$ so that the solution $W=W_{p_{1}, p_{2} ; c}$ of (3.7) with boundary 
conditions $W_{p_{1}, p_{2} ; c}(p)=W_{p_{1}, p_{2} ; c}\left(p_{2}\right)=0$ satisfies

$$
\left\{\left\|W_{p_{1}, p_{2} ; c}\right\|_{\infty} ;\left\|\nabla_{T_{c}} W_{p_{1}, p_{2} ; c}\right\|_{\infty}\right\}<K_{p_{1}, p_{2} ; c} \int_{0}^{\theta}\|\vec{\Psi}\| d s
$$

Now, the bounds $K_{p_{1}, p_{2} ; c}$ are determined by the coefficients of the homogeneous equation associated to (3.8), so after possibly replacing $K_{p_{1}, p_{2} ; c}$ with $4 \pi K_{p_{1}, p_{2} ; c}$ we conclude that for all pairs of points $x=\left(q_{1}, q_{2}\right) \in X_{0}$ near $\left(p_{1}, p_{2}\right) \in X_{0}$ and $t$ near $c$, then

$$
\left\{\left\|W_{x ; t}\right\|_{\infty} ;\left\|\nabla_{T_{t}}^{\widehat{\rho}_{t}} W_{x ; t}\right\|_{\infty}\right\} \leq K_{p_{1}, p_{2} ; c}\|\Psi\|_{\infty}
$$

Upon choosing a finite cover of $X_{0} \times\left[-T_{0} / 2, T_{0} / 2\right]$ by neighborhoods of $\left(x_{i}, c_{i}\right)$ $\in X_{0} \times\left[-T_{0} / 2, T_{0} / 2\right]$, we find the bound

$$
\left\{\left\|W_{t}\right\|_{\infty} ;\left\|\nabla_{T_{t}}^{\widehat{\rho}_{t}} W_{t}\right\|_{\infty}\right\} \leq K_{0}
$$

for all solutions to (3.6) along $\widehat{\rho}_{t}$-geodesic arcs $A_{t}$, and for all $t \in\left[-T_{0} / 2, T_{0} / 2\right]$.

We turn finally to using our estimate (3.9) to bound $l_{\widehat{\rho}_{\epsilon}}\left(A_{\epsilon}\right)$ from below for $\epsilon$ sufficiently small, where $A_{\epsilon}$ is the portion of the minimal $\widehat{\rho}_{\epsilon}$-geodesic $\gamma_{\epsilon}$ constructed at the outset of the argument. Because $l_{\widehat{\rho}_{t}}\left(A_{t}\right)$ is differentiable in $t$, we have the expansion

$$
l_{\widehat{\rho}_{\epsilon}}\left(A_{\epsilon}\right)=l_{\rho_{0}}\left(A_{0}\right)+\left.\epsilon \frac{d}{d t}\right|_{t=0}\left[l_{\widehat{\rho}_{t}}\left(A_{t}\right)\right]+\left.\frac{\epsilon^{2}}{2} \frac{d^{2}}{d t^{2}}\right|_{t=c}\left[l_{\widehat{\rho}_{t}}\left(A_{t}\right)\right]
$$

for some $c \in(0, \epsilon)$. In order to facilitate the evaluation of the derivatives it is convenient to introduce the following function of two variables

$$
l(t, w)=l_{\widehat{\rho}_{t}}\left(A_{w}\right)=\int_{A_{w}} \widehat{\rho}_{t}|d z|,
$$

where the first variable $t \in\left[-T_{0} / 2, T_{0} / 2\right]$ labels the different metrics, and the second variable $w \in\left[-T_{0} / 2, T_{0} / 2\right]$ the different arcs. Clearly we have $l_{\widehat{\rho}_{t}}\left(A_{t}\right)=$ 
$l(t, t)$ and therefore we simply have that the terms entering equation (3.10) are given by

$$
\frac{d}{d t} l(t, t)=\left.\frac{\partial}{\partial t} l(t, w)\right|_{w=t}+\left.\frac{\partial}{\partial w} l(t, w)\right|_{w=t}
$$

and,

$$
\frac{d^{2}}{d t^{2}} l(t, t)=\left.\frac{\partial^{2}}{\partial t^{2}} l(t, w)\right|_{w=t}+\left.2 \frac{\partial}{\partial t} \frac{\partial}{\partial w} l(t, w)\right|_{w=t}+\left.\frac{\partial^{2}}{\partial w^{2}} l(t, w)\right|_{w=t}
$$

where we have used the fact that the partial derivatives commute. Consider now the second term in the right hand side of (3.12). It corresponds to a first length variation for a family of curves; since the derivative is evaluated at $w=t$ and the corresponding curve $A_{t}$ is a geodesic in the metric $\widehat{\rho}_{t}$, this term vanishes identically for all $t$ :

$$
\left.\frac{\partial}{\partial w} l(t, w)\right|_{w=t} \equiv 0, \quad \text { for } t \in[-\epsilon, \epsilon]
$$

Taking a $t$ derivative of this equation

$$
\frac{d}{d t}\left(\left.\frac{\partial}{\partial w} l(t, w)\right|_{w=t}\right)=0, \quad \text { for } t \in[-\epsilon, \epsilon] .
$$

we obtain the following relation

$$
\left.\frac{\partial}{\partial t} \frac{\partial}{\partial w} l(t, w)\right|_{w=t}=-\left.\frac{\partial^{2}}{\partial w^{2}} l(t, w)\right|_{w=t} .
$$

Using (3.14) and (3.16), equations (3.12) and (3.13) reduce to

$$
\begin{aligned}
\frac{d}{d t} l(t, t) & =\left.\frac{\partial}{\partial t} l(t, w)\right|_{w=t}=\int_{A_{t}} h|d z|, \\
\frac{d^{2}}{d t^{2}} l(t, t) & =\left.\frac{\partial^{2}}{\partial t^{2}} l(t, w)\right|_{w=t}-\left.\frac{\partial^{2}}{\partial w^{2}} l(t, w)\right|_{w=t}=-\left.\frac{\partial^{2}}{\partial w^{2}} l(t, w)\right|_{w=t},
\end{aligned}
$$

where we used (3.11) with $\widehat{\rho}_{t}=\rho_{0}+t h$, so that $\frac{\partial^{2}}{\partial t^{2}} \widehat{\rho}_{t}=0$. We now claim that

$$
\left|\frac{\partial^{2}}{\partial w^{2}} l(t, w)\right|_{w=t} \mid<2 K
$$

where $K$ is a bound independent of the endpoints of the geodesic and of the value of 
$t \in(-\epsilon, \epsilon)$. This follows because the above is precisely the second length variation for a family of curves, evaluated for a base curve which is a geodesic. Using the standard formula (see Hicks [Hi] Chapter 10, Corollary, p. 151)

$\left.\frac{\partial^{2}}{\partial w^{2}} l(t, w)\right|_{w=t}=\int_{0}^{l\left(A_{t}\right)} d \sigma\left(<R(W, T) W, T>+<\nabla_{T} W, \nabla_{T} W>-(T<W, T>)^{2}\right)$,

we see that the boundedness of curvature, together with the uniform bounds of equation (3.9) guarantee the bound in (3.18).

Using equations (3.17) and (3.18) we find that equation (3.10) gives

$$
l_{\widehat{\rho}_{\epsilon}}\left(A_{\epsilon}\right)>l_{\rho_{0}}\left(A_{0}\right)+\int_{A_{0}} h|d z|-K \epsilon^{2}
$$

This inequality refers to an arc $A_{0} \subset \gamma_{0}$ running from $p_{1}$ to $p_{2}$ along $\gamma_{0}$. There is a similar inequality for the other subarc $A_{0}^{\prime} \subset \gamma_{0}$ continuing along $\gamma_{0}$ from $p_{2}$ back to $p_{1}$ (see Fig. 1). Adding the two we find

$$
l_{\widehat{\rho}_{\epsilon}}\left(\gamma_{\epsilon}\right)>l_{\rho_{0}}\left(\gamma_{0}\right)+\int_{\gamma_{0}} h|d z|-2 K \epsilon^{2} \geq 2 \pi-2 K \epsilon^{2}
$$

using hypothesis $(v)$ and the fact that $\gamma_{0}$ is a saturating geodesic.

Thus, in the metric $\rho_{\epsilon}=\widehat{\rho}_{\epsilon}+\frac{1}{\pi} K \epsilon^{2} \chi$, for $\epsilon$ sufficiently small, the shortest curve $\gamma_{\epsilon}$ will have length

$$
\begin{aligned}
l_{\rho_{\epsilon}}\left(\gamma_{\epsilon}\right) & =l_{\widehat{\rho}_{\epsilon}}\left(\gamma_{\epsilon}\right)+\frac{1}{\pi} K \epsilon^{2} l_{\rho_{0}}\left(\gamma_{\epsilon}\right) \\
& >2 \pi-2 K \epsilon^{2}+\frac{1}{\pi} K \epsilon^{2}(2 \pi)=2 \pi,
\end{aligned}
$$

where we have used that the admissibility of $\rho_{0}$ forces $l_{\rho_{0}}\left(\gamma_{\epsilon}\right) \geq 2 \pi$ and that $\gamma_{\epsilon} \subset \operatorname{supp} \chi$. Thus $\rho_{\epsilon}$ is admissible. This concludes our proof of the lemma. 
Consider now a surface with a metric $\rho_{0}$ of finite (reduced) area $A\left(\rho_{0}\right)$. The (reduced) area $A\left(\rho_{\epsilon}\right)$ of the surface with the $\rho_{\epsilon}$ metric defined in (3.1) is given by

$$
\begin{aligned}
A\left(\rho_{\epsilon}\right)= & A\left(\rho_{0}\right)+2 \epsilon \int h \rho_{0} d x d y+\epsilon^{2} \int\left(2 K \chi \rho_{0}+h^{2}\right) d x d y \\
& +2 \epsilon^{3} K \int h \chi d x d y+\epsilon^{4} K^{2} \int \chi^{2} d x d y
\end{aligned}
$$

This result is obvious when there are no punctures and the $A$ 's denote (ordinary) area. It holds when there are punctures, and we use reduced area, because the metric $\rho_{0}$ is complete and therefore the (compact) supports of $h$ and $\chi$ avoid some neighborhoods of the punctures. The $\mathcal{O}(\epsilon)$ and $\mathcal{O}\left(\epsilon^{3}\right)$ terms above are necessarily finite, since both $\rho_{0}$ and $h$ are smooth over the support of $h$. The $\mathcal{O}\left(\epsilon^{2}\right)$ and $\mathcal{O}\left(\epsilon^{4}\right)$ terms are also finite since $\rho_{0}$ must be square integrable over the support of $\chi$ (otherwise its (reduced) area would be infinite). Then, if the following inequality holds

$$
\int h \rho_{0} d x d y<0,
$$

we have that $A\left(\rho_{\epsilon}\right)<A\left(\rho_{0}\right)$ for sufficiently small $\epsilon$. If $\rho_{0}$ and $h$ satisfy the conditions of Lemma 3.1, the metric $\rho_{\epsilon}$ is admissible for sufficiently small $\epsilon$, and then (3.22) implies that $\rho_{0}$ cannot be of minimal area. We have therefore established the following corollary:

Corollary 3.2. Given a metric $\rho_{0}$ of finite (reduced) area and a smooth variation $h$ satisfying the conditions of Lemma 3.1, then $\int h \rho_{0} d x d y<0$ implies that the metric $\rho_{0}$ is not of minimal area.

Remark. Lemma 3.1 together with Corollary 3.2 give a partial local converse to Beurling's criterion [Ah]. In Beurling's criterion a sufficient (but not necessary) condition for extremal metrics is given. It involves showing that for any $h$ satisfying condition $(v)$ one must always be able to prove that the right hand side of (3.22) is greater or equal to zero. Our result is a partial converse because we have shown that for suitable $h$ and $\rho$ Beurling's condition is necessary. It is a local result because it tests locally whether the area of a metric can be reduced. 


\section{Neighborhoods of the Punctures are Flat}

In this section, we discuss the geometry of the minimal area metric in neighborhoods of the punctures of our punctured Riemann surface $\left(\mathcal{R} ; p_{1}, \ldots, p_{n}\right)$. In particular, we will show that these neighborhoods are isometric to flat cylinders of circumference $2 \pi$, foliated by a parallel family of geodesics of length $2 \pi$.

The idea will be to apply Corollary 3.2 to the case of a complete minimal area metric which is smooth in the neighborhood of a puncture. We show that if the metric is not flat, then it admits a deformation $h$ (as in Lemma 3.1) which lowers its area.

Theorem 4.1. Let $\rho_{0}$ be a complete minimal area metric on a Riemann surface $\mathcal{R}$ with punctures $p_{1}, \ldots, p_{n}$. Suppose that each puncture $p_{i}$ is contained in a neighborhood $N_{i}$ in which $\rho_{0}$ is smooth and non-vanishing. Then there are neighborhoods $V_{i}$ around $p_{i}$ in which $\rho_{0}$ is isometric to a flat semiinfinite cylinder of circumference $2 \pi$.

Proof. Consider a puncture $p$ surrounded by a neighborhood $N$ in which $\rho_{0}$ is smooth and non-vanishing. Lemma 2.1. implies that there is a neighborhood $V$ foliated by geodesics of length $2 \pi$, each homotopic to the puncture $p$. We claim that the leaves of the foliation are parallel, in the sense that if $\gamma_{1}(s)$ and $\gamma_{1}(\sigma)$ are two distinct leaves of the foliation, then $\min _{\sigma} d_{\rho_{0}}\left(\gamma_{1}(s), \gamma_{2}(\sigma)\right)=\min _{s, \sigma} d_{\rho_{0}}\left(\gamma_{1}(s), \gamma_{2}(\sigma)\right)$. To argue for this and the conclusion of the theorem, we work on the infinitesimal level, and consider the leaves of the foliation near a leaf $\gamma(0)$ as a variation of curves $\gamma(t)$. In particular, near $\gamma(0)$, we parametrize each leaf $\gamma(t)$ by arclength "s" so that $\gamma(t)=\gamma(s, t)$ and we claim that the variation field $\frac{\partial}{\partial t} \gamma(s, t)=V(s)$ is constant along $\gamma(0, s)$.

To see this consider a point $q \in \gamma(s, 0)$ at which $\frac{\partial}{\partial s}\|V(s)\|_{\rho_{0}}=\alpha \neq 0$; we will take $\alpha<0$.

We seek a more convenient parametrization of $\gamma(s, t)$. To this end, consider the vector field $X_{0}(s, t)$ of $\rho_{0}$-unit vectors which are perpendicular to the curves 
$\gamma(t)$. Since the $\rho_{0}$ metric is smooth, the vector field $X(s, t)$ is smooth and hence integrable; let the integral curve normal to $\gamma(\cdot, 0)$ at $\gamma(s, 0)$ be denoted by $N(s)$. We now parametrize $\gamma(s, t)$ so that $N(s)$ intersects $\gamma(\cdot, t)$ at $\gamma(s, t)$. Then, by Gauss' lemma, $\gamma(s, t)$ is still a parametrization by arclength for each fixed $t$. We normalize the parametrization by taking $q=\gamma(0,0)$ and assuming that the curve $N(0)$ is parametrized by arclength by the variable $t$ (see Fig. 2). Considering $\gamma(s, t)$ as a map from $[0,2 \pi] \times(-\epsilon, \epsilon)$ to $\mathcal{R}$ we set $X(s, t)=\left.\gamma_{*} \frac{\partial}{\partial t}\right|_{s, t}$; then $V(s)=X(s, 0)$. While $\|X(0, t)\|=1$ because the curve $N(0)$ is parametrized by arclength, $\|X(s, t)\|$ is not a priori of unit length. At the point $q$ we have assumed $\frac{\partial}{\partial s}\|V(s)\|<0$ (at $s=0)$.

Let $\Gamma_{-}$denote the subarc of $N(-\eta)$ with endpoints $\gamma(-\eta, 0)$ and $\gamma(-\eta, \kappa)$ and let $\Gamma_{+}$denote the subarc of $N(\eta)$ with endpoints $\gamma(\eta, 0)$ and $\gamma(\eta, \kappa)$. Roughly our plan is to exploit the fact that the length of $\Gamma_{-}$is bigger than that of $\Gamma_{+}$ since the norm of the variation field has negative derivative. Let $R_{-}$denote the intersection of the $\rho_{0}$-neighborhood of $\Gamma_{-}$of size $\delta$ with $\bigcup_{0 \leq t \leq \kappa} \gamma(\cdot, t)$. We observe that since the leaves of the foliation are perpendicular to $\Gamma_{-}$, then $R_{-}$can also be described as the union of arcs of the foliation of $\rho_{0}$-length $2 \delta$ centered along $\Gamma_{-}$. We similarly define $R_{+}$as the intersection of the $\rho_{0}$-neighborhood of $\Gamma_{+}$of size $\delta$ with $\bigcup_{0 \leq t \leq \kappa} \gamma(\cdot, t)$ (see Fig. 2).

Let $k$ and $k^{\prime}$ be smooth positive functions supported on the intervals $(-\delta, \delta)$ and $(0, \kappa)$ respectively. Consider then the smooth positive function $K: \mathbf{R}^{2} \rightarrow \mathbf{R}$, defined as $K(s, t)=k(s) k^{\prime}(t)$. This function is compactly supported in a rectangle. We use $K$ to define the smooth (metric) $h$ that we need; $h$ will be supported on $R_{-} \cup R_{+}$. For any point $q$ with coordinates $(s(q), t(q))$ we define $h$ by

$$
\begin{aligned}
& h(q)=-\rho_{0} K(s(q)+\eta, t(q)) \quad \text { for } q \in R_{-}, \\
& h(q)=\rho_{0} K(s(q)-\eta, t(q)) \quad \text { for } q \in R_{+}, \\
& h(q)=0, \quad \text { elsewhere. }
\end{aligned}
$$

Consider the metric $\widehat{\rho}_{\epsilon}=\rho_{0}+\epsilon h$. Conditions $(i)-(i v)$ of Lemma 3.1 are satisfied 
so we now verify condition $(v)$. For any saturating geodesic $\gamma(t)$ one should have $\int_{\gamma(t)} h|d z| \geq 0$; since $\rho_{0}|d z|$ is the length element $d s$ we have

$$
\begin{aligned}
\int_{\gamma(t)} h|d z|=\int_{\gamma(t)} \frac{h}{\rho_{0}} d s & =\int_{-\eta-\delta}^{-\eta+\delta} \frac{h(s, t)}{\rho_{0}} d s+\int_{\eta-\delta}^{\eta+\delta} \frac{h(s, t)}{\rho_{0}} d s \\
& =-\int_{-\eta-\delta}^{-\eta+\delta} K(s+\eta, t) d s+\int_{\eta-\delta}^{\eta+\delta} K(s-\eta, t) d s \\
& =-\int_{-\delta}^{\delta} K(s, t) d s+\int_{-\delta}^{\delta} K(s, t) d s=0
\end{aligned}
$$

thus our deformation precisely preserves the length of the saturating geodesics. Since $h$ satisfies all the conditions of Lemma 3.1 we can now apply Corollary 3.1 to show that the metric cannot be of minimal area. We must simply verify that the quantity indicated in (3.22) is negative:

$$
\iint_{R_{-} \cup R_{+}} h \rho_{0} d x d y=\iint_{R_{-} \cup R_{+}} \frac{h}{\rho_{0}} d A\left(\rho_{0}\right)<0
$$

The left hand side of the inequality is given explicitly by

$$
\int_{0}^{\kappa} \int_{-\eta-\delta}^{-\eta+\delta} \frac{h(s, t)}{\rho_{0}}\left\|\gamma_{*} \frac{\partial}{\partial s}\right\|_{\rho_{0}}\left\|\gamma_{*} \frac{\partial}{\partial t}\right\|_{\rho_{0}} d s d t+\int_{0}^{\kappa} \int_{\eta-\delta}^{\eta+\delta} \frac{h(s, t)}{\rho_{0}}\left\|\gamma_{*} \frac{\partial}{\partial s}\right\|_{\rho_{0}}\left\|\gamma_{*} \frac{\partial}{\partial t}\right\|_{\rho_{0}} d s d t
$$

since the $(s, t)$ coordinate system is orthogonal. Using $\left\|\gamma_{*} \frac{\partial}{\partial s}\right\|_{\rho_{0}}=1$ (parametrization is by arclength) and $\left\|\gamma_{*} \frac{\partial}{\partial t}\right\|_{\rho_{0}}=\|X(s, t)\|$, we find that (4.4) becomes

$$
-\int_{0}^{\kappa} \int_{-\eta-\delta}^{-\eta+\delta} K(s+\eta, t)\|X(s, t)\| d s d t+\int_{0}^{\kappa} \int_{\eta-\delta}^{\eta+\delta} K(s-\eta, t)\|X(s, t)\| d s d t
$$


and finally, shifting the domains of integration we obtain

$$
-\int_{0}^{\kappa} \int_{-\delta}^{\delta} K(s, t) g(s, t) d s d t, \quad \text { where } \quad g(s, t)=\|X(-\eta+s, t)\|-\|X(\eta+s, t)\|
$$

Since $g(0,0)=\|V(-\eta)\|-\|V(\eta)\|$, and, by assumption, $\frac{\partial}{\partial s}\|V(s)\|<0$ for $s=0$, for sufficiently small $\eta$ one must have $g(0,0)>0$. Since $g$ is continuous, it follows that it is positive throughout the region of integration for sufficiently small $\delta$ and $\kappa$. It is then clear that the above expression is strictly negative, as we wanted to show. This proves that $\frac{\partial}{\partial s}\|V(s)\|=0$, at $q \in \gamma(0)$ (corresponding to $s=0$ ). Since this point is not a special point, it follows that $\frac{\partial}{\partial s}\|V(s)\|=0$, for all points in the geodesic $\gamma(0)$. Since the geodesic $\gamma(0)$ is not a special geodesic, we have $\frac{\partial}{\partial s}\|X(s, t)\|=0$ along every leaf of the foliation. Since $\|X(0, t)\|=1$, we have shown that $\|X(s, t)\|=\left\|\gamma_{*} \frac{\partial}{\partial t}\right\|=1$.

Finally, we consider the map $\gamma(s, t)$ as a map from a flat cylinder $C$ with locally Euclidean metric $\bar{\rho}=d s^{2}+d t^{2}$ to the neighborhood $V$ of the puncture with metric $\rho_{0}$. The map $\gamma(s, t)$ is a (local) diffeomorphism, and so we consider the pullback metric $\gamma(s, t)^{*} \rho_{0}$ as a smooth metric on the cylinder $C$. In comparing the pullback metric $\gamma(s, t)^{*} \rho_{0}$ with the flat metric $\bar{\rho}$, we first notice that both $\gamma_{*} \frac{\partial}{\partial s}$ and $\gamma_{*} \frac{\partial}{\partial t}$ are of unit $\rho_{0}$-length and orthogonal for all $s$ and $t$ : we conclude that $\gamma(s, t)^{*} \rho_{0}=\bar{\rho}$ and so $\left(V, \rho_{0}\right)$ is isometric to a flat annulus. This concludes the proof of Theorem 4.1.

Remark. One often shows that for extremal length problems, the neighborhoods in which the metric is smooth must be flat (see [St] for example), and it is common to use the length-area method in such arguments. Of course, the length-area method requires an analysis along a neighborhood of an entire geodesic; in the above argument, however, the analysis was restricted to a neighborhood of a point, after requiring some smoothness and finiteness properties of the neighborhood of the geodesic. 


\section{Amputation and Plumbing of Minimal Area Surfaces}

The purpose of the present section is to give a proof of the amputation property of generalized minimal area metrics on punctured surfaces. This will be done in section $\S 5.1$. In $\S 5.2$ we will discuss, for completeness of exposition, the plumbing of minimal area metrics. Expanding the discussion of $[\mathrm{Zw} 2]$ we show that the plumbing of surfaces with metrics of minimal area, using the canonical coordinates induced by these metrics, induces on the resulting surface a minimal area metric.

Before beginning our exposition let us establish a useful lower bound on lengths of nontrivial closed curves. Consider a Riemann surface with an admissible metric and a ring domain $F_{i}$ on the surface isometric to a flat cylinder (finite or semiinfinite) of circumference $2 \pi$. This ring is foliated by saturating geodesics homotopic to its core curve. Denote one of the boundaries of the ring domain by $\mathcal{C}_{0}$, and let the curve $\mathcal{C}_{\delta}$ denote the saturating geodesic at a distance $\delta$ away from $\mathcal{C}_{0}$. Let $\mathcal{C}_{h}$ denote the other boundary of the ring domain; $h$ is called the height of the cylinder ( $h=\infty$, if $F_{i}$ is a semiinfinite cylinder). We say that a nontrivial closed curve $\gamma$ penetrates $F_{i}$ a distance $\delta(\delta<h)$ if one can find two points $p_{1}, p_{2} \in\left(\gamma \cap \mathcal{C}_{0}\right)$ and an open subcurve $\gamma^{\prime}$ of $\gamma$ with endpoints $p_{1}$ and $p_{2}$, fully contained in $F_{i}$, such that $\gamma^{\prime} \cap \mathcal{C}_{\delta} \neq 0$, and $\gamma^{\prime} \cap \mathcal{C}_{\eta}=0$, for $h>\eta>\delta$.

We say that a nontrivial closed curve $\gamma$ extends a distance $\delta$ in a ring domain $F_{i}$ (as above) if the curve is fully contained in $F_{i}$, it is homotopic to the core curve in the ring, and there are two unique saturating curves $\mathcal{C}_{\eta}$ and $\mathcal{C}_{\eta+\delta}$ in $F_{i}$ such that $\gamma \cap \mathcal{C}_{\eta} \neq 0$, and $\gamma \cap \mathcal{C}_{\eta+\delta} \neq 0$.

Lemma 5.1. Consider a surface with an admissible metric and a nontrivial closed curve $\gamma$ that penetrates a ring domain $F_{i}$, isometric to a flat cylinder of circumference $2 \pi$, a distance $\delta$. The length $l(\gamma)$ of the curve $\gamma$ satisfies the inequality

$$
l(\gamma) \geq 2 \pi \sqrt{1+\delta^{2} / \pi^{2}} .
$$

The same inequality holds for a nontrivial closed curve $\gamma$ which extends a distance $\delta$ on $F_{i}$. 
Proof. Consider a curve $\gamma$ penetrating $F_{i}$ a distance $\delta$. Then the two points $p_{1}$ and $p_{2}$ determine a segment $\overline{p_{1} p_{2}}$ on $\mathcal{C}_{0}$ homotopic to the subcurve $\gamma^{\prime}$ lying completely on $F_{i}$ whose endpoints are $p_{1}$ and $p_{2}$. Let $d$ denote the length on the (extension of the) flat metric on the cylinder of the segment $\overline{p_{1} p_{2}}$. It is clear from the flat geometry of the cylinder (see Fig. 3) that the length $l\left(\gamma^{\prime}\right)$ must exceed that of the segment $\overline{p_{1} p_{2}}$ by

$$
l\left(\gamma^{\prime}\right)-l\left(\overline{p_{1} p_{2}}\right) \equiv e(d) \geq 2 \sqrt{\delta^{2}+d^{2} / 4}-d
$$

Since the metric is admissible and the subcurve $\gamma^{\prime}$ is homotopic to the segment $\overline{p_{1} p_{2}}$, it follows that $l(\gamma) \geq 2 \pi+e(d)$, where $e(d)$ is the excess length defined above. The function $e(d)$ can be readily checked to be monotonically decreasing in $d$. It will therefore attain its minimum value for the maximum possible value of $d$, namely $2 \pi$. We then have

$$
l(\gamma) \geq 2 \pi+e(2 \pi)=2 \pi \sqrt{1+\delta^{2} / \pi^{2}}
$$

This is the desired bound. The computation for the case of a curve that extends a distance $\delta$ in the flat cylinder $F_{i}$ is enterely analogous, and the conclusion is the same.

\subsection{Proof of Amputation}

Consider a punctured Riemann surface $\mathcal{R}$ equipped with a complete metric $\rho_{0}$ smooth near the punctures, and solving the generalized minimal area problem. We have shown that around each puncture $p_{i}$ there is a neighborhood where the metric is that of a flat semiinfinite cylinder of circumference $2 \pi$.

Let us now use this to find a canonical domain near $p_{i}$ on which to perform the amputation. Consider the set $\mathcal{U}_{i}^{*}=\{p \in \mathcal{R} \mid$ there exists exactly one nontrivial curve $\gamma_{p}$ through $p$ of length $2 \pi$. The curve $\gamma_{p}$ is homotopic to $p_{i}$, and $\rho_{0}$ is smooth near $p$. Let $\mathcal{U}_{i}$ denote the connected component of $\mathcal{U}_{i}^{*}$ which meets every 
neighborhood of $p_{i}$; the domain $\mathcal{U}_{i}$ is foliated by geodesics homotopic to $p_{i}$, and is conformally equivalent to the punctured disk $\left\{\left|\zeta_{i}\right|<1\right\}$. This disk $\mathcal{U}_{i}$ with metric $\rho_{0}$ is isometric to a flat semiinfinite cylinder of circumference $2 \pi$ foliated by saturating geodesics homotopic to $p_{i}$. It is the maximal such cylinder around the puncture. The saturating geodesics are the curves of constant $|\zeta|$. The disk $\mathcal{U}_{i}$ is the canonical domain defined by the minimal area metric.

From now on let us consider a single puncture $p$, and its corresponding maximal $\operatorname{disk} \mathcal{U}(|\zeta|<1)$. Define on $\mathcal{U}$ the coordinate $t=-\log \zeta$, and let $\mathcal{C}_{\delta}$ with $\delta \in[0, \infty]$ denote the saturating geodesic $|t|=\delta$. The curve $\mathcal{C}_{0}$ is therefore the boundary of $\mathcal{U}$, and $\delta$ measures the $\rho_{0}$-distance between $\mathcal{C}_{\delta}$ and $\mathcal{C}_{0}$. The curve $\mathcal{C}_{\delta}$ divides the surface $\mathcal{R}$ into two pieces: the subdisk $\mathcal{U}_{\delta}\left(\equiv \mathcal{U}-\left\{|\zeta| \geq e^{-\delta}\right\}\right)$ and the amputated surface $\mathcal{R}_{\delta}\left(\equiv \mathcal{R}-\mathcal{U}_{\delta}\right)$. Finally we define, for a fixed $\delta \neq 0$ the "stub" $\mathcal{S}_{\delta}$ to be the annulus $\mathcal{U}-\mathcal{U}_{\delta}$. Clearly $\mathcal{R}_{\delta}=\mathcal{R}_{0} \cup \mathcal{S}_{\delta}$ (see Fig. 4).

Theorem 5.1. (Amputation) Consider a surface $\mathcal{R}$ with a minimal area metric $\rho_{0}$ defining a canonical domain around a puncture $p$. Assume the amputated surface $\mathcal{R}_{\delta}$ has a minimal area metric $\rho$ continuous in a neighborhood of the boundary $\mathcal{C}_{\delta}$. Then $\rho$ is the restriction of $\rho_{0}$ to $\mathcal{R}_{\delta}$.

Proof. Our proof will involve several steps. We will first assume there is a candidate metric on the amputated surface having less area. The obvious idea, which is to use the candidate metric on the amputated surface, together with the original metric on the remainder of $\mathcal{R}$ to define an admissible metric on the whole surface, does not work because the resulting metric would be discontinuous on the cutting line and bad curves could appear. Nevertheless, with a careful treatment of the neighborhood of the cutting line we will succeed in constructing an admissible metric on $\mathcal{R}$ of lower area that that of $\rho_{0}$.

We begin by assuming Theorem 5.1 does not hold and there is another metric $\rho_{1}$ on $\mathcal{R}_{\delta}$ continuous on a neighborhood of $\mathcal{C}_{\delta}$, satisfying all the length conditions on $\mathcal{R}_{\delta}$ and having less area than $\rho_{0}$. Note that $\rho_{1} \neq \alpha \rho_{0}$, where $\alpha$ is a constant, since 
the lower area condition would imply $\alpha<1$, and this would make the geodesics in the stub $\mathcal{S}_{\delta}$ shorter than $2 \pi$.

Our aim will be to construct, using $\rho_{0}$ and $\rho_{1}$ a candidate metric on $\mathcal{R}_{\delta}$, of area lower than $\rho_{0}$, which can be glued back to the cylinder representing the puncture giving an admissible metric on $\mathcal{R}$. This would be a contradiction since it would show that $\rho_{0}$ was not the minimal area metric on $\mathcal{R}$. Begin by considering the family $\rho_{\epsilon}$ of metrics on $\mathcal{R}_{\delta}$ given by

$$
\rho_{\epsilon}=(1-\epsilon) \rho_{0}+\epsilon \rho_{1}
$$

where $0<\epsilon<1$. It is not difficult to see that $\rho_{0}$ is an admissible metric on $\mathcal{R}_{\delta}$ (see the proof of Corollary 5.7 below), and, by hypothesis, the metric $\rho_{1}$ is admissible, so, by linearity, we see that $\rho_{\epsilon}$ is an admissible metric on $\mathcal{R}_{\delta}$. Let $A_{0}$ and $A_{1}$ denote the area of $\mathcal{R}_{\delta}$ in the $\rho_{0}$ and $\rho_{1}$ metrics respectively. We now calculate the area $A_{\epsilon}$ of $\mathcal{R}_{\delta}$ using the metric $\rho_{\epsilon}$. It follows from Eqn. (5.4) that

$$
A_{\epsilon}=(1-\epsilon)^{2} A_{0}+2(1-\epsilon) \epsilon \int_{\mathcal{R}_{\delta}} d^{2} \xi \rho_{0} \rho_{1}+\epsilon^{2} A_{1}
$$

Since we know that $\rho_{1} \neq \alpha \rho_{0}$ ( $\alpha$ a constant), the Schwarz inequality gives us

$$
\int_{\mathcal{R}_{\delta}} d^{2} \xi \rho_{0} \rho_{1}=\sqrt{A_{0} A_{1}}-\Delta
$$

with $\Delta>0$, since the Schwarz inequality is not saturated. It follows from Eqns. (5.5) and (5.6) that

$$
A_{\epsilon}=A_{0}-\left|\beta_{1}\right| \epsilon+\left|\beta_{2}\right| \epsilon^{2}
$$

where the constants $\left|\beta_{1}\right|$ and $\left|\beta_{2}\right|$ are both strictly positive and given by

$$
\left|\beta_{1}\right|=2\left(A_{0}\left(1-\sqrt{\frac{A_{1}}{A_{0}}}\right)+\Delta\right)>0, \quad\left|\beta_{2}\right|=\left(\sqrt{A_{0}}-\sqrt{A_{1}}\right)^{2}+2 \Delta>0 .
$$

It follows from Eq. (5.7), that for sufficiently small $\epsilon$ the area $A_{\epsilon}$ is strictly smaller than $A_{0}$. 
We will work throughout with the canonical local coordinates determined by the metric $\rho_{0}$; in these coordinates $\rho_{0}=1$ on the cylinder. Consider a neighborhood $\mathcal{N}(r) \subset \mathcal{R}_{\delta}$ of the boundary $\mathcal{C}_{\delta}$ consisting of all points in $\mathcal{R}_{\delta}$ whose $\rho_{0}$-distance to $\mathcal{C}_{\delta}$ is smaller than $r$. Choose $\epsilon$, such that $\epsilon^{1 / 4}<\delta$; therefore the neighborhood $\mathcal{N}\left(\epsilon^{1 / 4}\right)$ is contained in the stub $\mathcal{S}_{\delta}$. Let the constant $K$ be defined by

$$
1+K \equiv \sup _{\mathcal{N}\left(\epsilon^{1 / 4}\right)} \rho_{1}
$$

where $\rho_{1}$ is the value of the conformal factor in the flat local coordinates defined by $\rho_{0}$. The constant $K$ is bounded since the candidate metric $\rho_{1}$ has been assumed to be continuous on a neighborhood of $\mathcal{C}_{\delta}$ and therefore, for sufficiently small $\epsilon$ equation (5.9) defines a finite $K$. It follows from Eq. (5.4) that

$$
\sup _{\mathcal{N}\left(\epsilon^{1 / 4}\right)} \rho_{\epsilon}=1+K \epsilon \equiv M(\epsilon)
$$

where we have defined $M(\epsilon)$ for later convenience.

Let us see that $K>0$. If $K<0$ then, $\rho_{1}<1$ throughout the neigborhood, but then it would not be admissible. If $K=0$, then $\rho_{1} \leq 1$ in the full neighborhood. Admissibility then requires $\rho_{1} \equiv 1$ and therefore $\rho_{1}=\rho_{0}$ in the neighborhood. The punctured disk could be restored at this stage, since the metric is continuous along the cutting curve, which is of length $2 \pi$, the full metric is admissible, and of lower area. This is a contradiction. Therefore $K>0$, and bounded, is the only case we need to consider.

Let us extend the definition of the metric $\rho_{1}$ to $\mathcal{R}$ by letting $\rho_{1}=\rho_{0}$ on $\mathcal{U}_{\delta}$. This metric is clearly discontinuous on $\mathcal{R}$ and may have bad curves. We quantify this possibility in the following lemma.

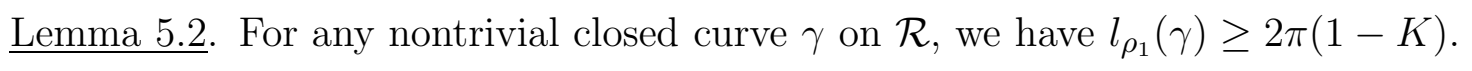

Proof. Any curve fully on $\mathcal{R}_{\delta}$ or $\mathcal{U}_{\delta}$ clearly satisfies the inequality (in fact they are both longer than $2 \pi$ ). The only problem are the curves that cross $\mathcal{C}_{\delta}$. Divide 
such a curve $\gamma$ into two pieces $\gamma_{\text {in }}$, which is the part of $\gamma$ on $\mathcal{R}_{\delta}$, and $\gamma_{\text {out }}$, which is the part of $\gamma$ on $\mathcal{U}_{\delta}$ (out of the amputated surface). The possibility that curves can be shorter than $2 \pi$ is due to the discontinuity of the metric $\rho_{1} \operatorname{across} \mathcal{C}_{\delta}$. The portion $\gamma_{\text {out }}$ is made of segments of lengths $b^{i}$, where the index $i$ labels the different segments. Each segment is homotopic to a segment $a_{i}$ on $\mathcal{C}_{\delta}$. The length of this segment $a_{i} \in \mathcal{C}_{\delta}$ depends on whether it is taken with respect to the metric $\rho_{1}$ in $\mathcal{R}_{\delta}$ (the in-metric) or with respect to the (extension of the metric in $\mathcal{U}_{\delta}$ to the) metric in $\overline{\mathcal{U}}_{\delta}$ (the out-metric). Let those lengths be be denoted by $a_{\text {in }}^{i}$ or $a_{\text {out }}^{i}$ respectively.

Since the maximum value of $\rho_{1}$ in a neighborhood of $\mathcal{C}_{\delta}$ lying on $R_{\delta}$ is $(1+K)$ (Eq. (5.9)), it follows that

$$
a_{\text {in }}^{i} \leq(1+K) a_{\text {out }}^{i} \quad \text { so that } \quad a_{\text {in }}^{i}-a_{\text {out }}^{i} \leq K a_{\text {out }}^{i}
$$

We now have that

$$
l_{\rho_{1}}(\gamma)=l_{\rho_{1}}\left(\gamma_{\text {in }}\right)+\sum b^{i} \geq l_{\rho_{1}}\left(\gamma_{\text {in }}\right)+\sum a_{\text {out }}^{i}
$$

where the last inequality follows from $b^{i} \geq a_{\text {out }}^{i}$, which follows because on $\mathcal{U}_{\delta}$ the metric $\rho_{1}$ is that of a flat cylinder, and therefore a segment of a core curve $\left(a_{\text {out }}^{i}\right)$ is shorter than any other homotopic open curve between its endpoints.

Let us now consider two cases. If $\sum a_{\text {out }}^{i} \geq 2 \pi$, then the above equation tells us we are done (the curve is longer than $2 \pi$ ). Let us now consider the case when $\sum a_{\text {out }}^{i} \leq 2 \pi$. It follows from Eq. (5.12) that

$$
l_{\rho_{1}}(\gamma) \geq l_{\rho_{1}}\left(\gamma_{i n}\right)+\sum a_{i n}^{i}-\sum\left(a_{\text {in }}^{i}-a_{\text {out }}^{i}\right)
$$

The first two terms in the right hand side give the length of a closed curve entirely on $\mathcal{R}_{\delta}$ and of the same homotopy type as the original curve. Since $\rho_{1}$ is admissible on $\mathcal{R}_{\delta}$ these two terms add up to $2 \pi$ or more. For the last term we use Eq. (5.11), 
and find

$$
l_{\rho_{1}}(\gamma) \geq 2 \pi-K \sum a_{\text {out }}^{i}
$$

Since we are considering the case when $\sum a_{\text {out }}^{i} \leq 2 \pi$, the above inequality reduces to $l_{\rho_{1}}(\gamma) \geq 2 \pi(1-K)$, which is the desired statement.

We now extend the definition of $\rho_{\epsilon}$ to the full surface $\mathcal{R}$ by letting $\rho_{\epsilon} \equiv \rho_{0}$ on $\mathcal{U}_{\delta}$. This is compatible with eqn.(5.4) and the definition of $\rho_{1}$ over $\mathcal{R}$. It is now possible to show that most curves are good for the metric $\rho_{\epsilon}$ on $\mathcal{R}$, where we recall that a $\rho_{\epsilon}$-good curve has $\rho_{\epsilon}$-length of at least $2 \pi$. This is the content of the following Lemma:

Lemma 5.3. The metric $\rho_{\epsilon}$ in $\mathcal{R}$ is admissible for all curves except for those completely contained in $\mathcal{N}\left(\epsilon^{1 / 3}\right) \cup \mathcal{U}_{\delta}$, and crossing the boundary curve $\mathcal{C}_{\delta}$.

Proof. Nontrivial closed curves are either homotopic to the puncture or are not. Let us begin by showing that the curves that are not homotopic to the puncture are always good. If such curve is completely contained in $\mathcal{R}_{\delta}$ it is clearly good (see below Eq. (5.4)). Consider then a curve $\gamma$ that extends into $\mathcal{U}_{\delta}$ (see Fig. 4). Since it completely penetrates the stub $\mathcal{S}_{\delta}$ we have (Lemma 5.1)

$$
l_{\rho_{0}}(\gamma) \geq 2 \pi \sqrt{1+\delta^{2} / \pi^{2}}
$$

and moreover, $l_{\rho_{1}}(\gamma) \geq 2 \pi(1-K)$ (Lemma 5.2). Therefore the equality

$$
l_{\rho_{\epsilon}}(\gamma)=(1-\epsilon) l_{\rho_{0}}(\gamma)+\epsilon l_{\rho_{1}}(\gamma)
$$

leads to the inequality

$$
l_{\rho_{\epsilon}}(\gamma) \geq(1-\epsilon) 2 \pi \sqrt{1+\delta^{2} / \pi^{2}}+\epsilon 2 \pi(1-K)
$$


It follows that $l_{\rho_{\epsilon}}(\gamma) \geq 2 \pi$ if we have

$$
(1-\epsilon)\left(\sqrt{1+\delta^{2} / \pi^{2}}-1\right)-K \epsilon \geq 0
$$

Taking $\epsilon<1 / 2$, it is then sufficient to require

$$
\sqrt{1+\delta^{2} / \pi^{2}}-1 \geq 2 K \epsilon \text { so that } \quad \frac{\delta^{2}}{4 \pi^{2}} \geq K \epsilon+(K \epsilon)^{2} .
$$

This is easily satisfied. If $\left(\delta^{2} / 4 \pi^{2}\right)<1$, we take $\epsilon<\delta^{2} / 8 K \pi^{2}$. If $\left(\delta^{2} / 4 \pi^{2}\right) \geq 1$, it is sufficient to take $\epsilon<1 / 2 K$. This shows that curves that are not homotopic to the puncture can be made to have $\rho_{\epsilon}$ lengths at least $2 \pi$ by choosing $\epsilon$ sufficiently small.

Now consider the curves homotopic to the puncture. If they lie completely on $\mathcal{R}_{\delta}$ or $\mathcal{U}_{\delta}$ they are good. By the statement of the lemma the only curves we need to discuss are those that go into the surface $\mathcal{R}_{\delta}$ beyond $\mathcal{N}\left(\epsilon^{1 / 3}\right)$ and also get into $\mathcal{U}_{\delta}$. We can easily give an estimate of the length of such curve in the $\rho_{0}$ metric. Since it penetrates a foliation for a distance $\epsilon^{1 / 3}$ we have that

$$
l_{\rho_{0}}(\gamma) \geq 2 \pi \sqrt{1+\epsilon^{2 / 3} / \pi^{2}}
$$

and using our estimate on $l_{\rho_{1}}(\gamma)$, we find

$$
l_{\rho_{\epsilon}}(\gamma) \geq(1-\epsilon) 2 \pi \sqrt{1+\epsilon^{2 / 3} / \pi^{2}}+\epsilon 2 \pi(1-K)
$$

Admissibility requires that (again $\epsilon<1 / 2$ )

$$
\sqrt{1+\epsilon^{2 / 3} / \pi^{2}} \geq 1+2 K \epsilon \text { so that } \frac{\epsilon^{2 / 3}}{4 \pi^{2}} \geq K \epsilon+(K \epsilon)^{2} .
$$

Since the left hand side of the last inequality is less than one, it is sufficient to take $K \epsilon<\epsilon^{2 / 3} / 8 \pi^{2}$, which just requires $\epsilon<1 /\left(8 \pi^{2}\right)^{3} K^{3}$. Thus for sufficiently small $\epsilon$ we have admissibility. This concludes our proof of lemma 5.3. 
We now need to improve the metric $\rho_{\epsilon}$ in order to get the remaining curves to have sufficient length. These are the curves that cross the boundary $\mathcal{C}_{\delta}$, and on $\mathcal{R}_{\delta}$ do not extend beyond a distance $\epsilon^{1 / 3}$ of $\mathcal{C}_{\delta}$. Define now on $\mathcal{R}_{\delta}$ a new metric $\rho_{\epsilon}{ }^{\prime}$ that is flat and constant near the curve $\mathcal{C}_{\delta}$ :

$$
\rho_{\epsilon}^{\prime}=\rho_{\epsilon}+f_{\epsilon}(r)\left(M(\epsilon)-\rho_{\epsilon}\right)
$$

where $M(\epsilon)$ was defined in (5.10), and $r$ denotes $\rho_{0}$-distance to $\mathcal{C}_{\delta}$. Here the function $f_{\epsilon}$ is an interpolating function whose value is $f_{\epsilon}(r)=1$ for $r<\epsilon^{1 / 3}$, and $f_{\epsilon}(r)=0$ for $r>\epsilon^{1 / 4}$. For $\epsilon^{1 / 3}<r<\epsilon^{1 / 4}$, the function $f_{\epsilon}$ is monotonically decreasing and continuous. Note that on $\mathcal{N}\left(\epsilon^{1 / 3}\right)$ we have $\rho_{\epsilon}{ }^{\prime}=M(\epsilon)=1+K \epsilon$, which is just a constant. On $\mathcal{U}_{\delta}$ we set the metric $\rho_{\epsilon}{ }^{\prime} \equiv \rho_{0}$.

Lemma 5.4. The metric $\rho_{\epsilon}^{\prime}$ is an admissible metric on $\mathcal{R}$, and its area, for sufficiently small $\epsilon$, is lower than that of $\rho_{0}$.

Proof. The metric $\rho_{\epsilon}{ }^{\prime}$ is admissible on $\mathcal{R}$ for the same curves $\rho_{\epsilon}$ was, since it differs from $\rho_{\epsilon}$ by the addition of a term that is always positive. The remaining curves, namely those that extend over the two domains $\mathcal{N}\left(\epsilon^{1 / 3}\right)$ and $\mathcal{U}_{\delta}$ only, now clearly are $\rho_{\epsilon}^{\prime}$-good. Since over all of $\mathcal{N}\left(\epsilon^{1 / 3}\right)$ the metric $\rho_{\epsilon}^{\prime}=1+K \epsilon>1=\rho_{0}$, any closed curve could only have grown in size with respect to its original total length in the $\rho_{0}$ metric. Thus any such curve must be longer or equal to $2 \pi$. The improvement term in Eq. (5.23) was necessary to get this type of curves to be $\rho_{\epsilon}^{\prime}$-good.

Let us now calculate the area $A_{\epsilon}^{\prime}$ of the metric $\rho_{\epsilon}{ }^{\prime}$ on $R_{\delta}$. It follows from Eq. (5.23) that:

$$
A_{\epsilon}^{\prime}=A_{\epsilon}+2 \int d^{2} \xi \rho_{\epsilon} f_{\epsilon}\left(M(\epsilon)-\rho_{\epsilon}\right)+\int d^{2} \xi f_{\epsilon}^{2}\left(M(\epsilon)-\rho_{\epsilon}\right)^{2} .
$$

Since $\operatorname{supp}\left(f_{\epsilon}\right) \subset \mathcal{N}\left(\epsilon^{1 / 4}\right)$, we see that we need only estimate these integrals over 
$\mathcal{N}\left(\epsilon^{1 / 4}\right)$. In this region we have the following inequalities

$$
f_{\epsilon}(r) \leq 1, \quad \rho_{\epsilon} \leq 1+K \epsilon, \quad M(\epsilon)-\rho_{\epsilon} \leq 1+K \epsilon-(1-\epsilon)=(K+1) \epsilon,
$$

where in the last one, we have used the fact that the lowest possible value of $\rho_{1}$ is zero. We can now estimate the integrals in Eq. (5.24) to find $\left(\int d^{2} \xi=2 \pi \int d r\right)$

$$
A^{\prime}{ }_{\epsilon} \leq A_{\epsilon}+4 \pi(1+K \epsilon)(1+K) \epsilon^{5 / 4}+2 \pi(1+K)^{2} \epsilon^{9 / 4}
$$

Taking $K \epsilon<1$, namely $\epsilon<1 / K$ and using Eq. (5.7), we have

$$
A^{\prime}{ }_{\epsilon} \leq A_{0}-\left|\beta_{1}\right| \epsilon+|\eta| \epsilon^{5 / 4}+\left|\beta_{2}\right| \epsilon^{2}+|\kappa| \epsilon^{9 / 4}
$$

where $|\eta|=8 \pi(1+K)$ and $|\kappa|=2 \pi(1+K)^{2}$. It is clear from Eq. (5.27) that for sufficiently small $\epsilon$ the area of $\rho_{\epsilon}{ }^{\prime}$ on $\mathcal{R}_{\delta}$ is lower than that of $\rho_{0}$ on $\mathcal{R}_{\delta}$. The same is therefore true for the area on the whole surface $\mathcal{R}$. This concludes our proof of lemma 5.4 .

Having established Lemma 5.4, the existence of a metric $\rho_{1}$ of lesser area than $\rho_{0}$ on $\mathcal{R}_{\delta}$ has enabled us to construct an admissible metric on $\mathcal{R}$ of area lower than that of the minimal area metric $\rho_{0}$. This contradiction establishes that the restriction of $\rho_{0}$ is indeed the minimal area metric on the amputated surface $\mathcal{R}_{\delta}$. This concludes our proof of Theorem 5.1.

\subsection{Plumbing Minimal Area Metrics}

We conclude this section by describing the minimal area metric on a surface obtained by the conformal plumbing of surfaces which admit minimal area metrics which are smooth and complete near the appropriate punctures. What follows is a detailed exposition of the ideas briefly sketched in [Zw2]. 
To begin, we recall from the introduction that the process of conformal plumbing begins either with a surface $\mathcal{R}_{0}$ with at least a pair of punctures, or two surfaces $\mathcal{R}_{0}^{\prime}$ and $\mathcal{R}_{0}^{\prime \prime}$ each with at least one puncture. In the first case, say, we consider small neighborhoods $U_{|t|, 1}=\left\{\zeta_{1}|| \zeta_{1}|<| t \mid\right\}$ and $U_{|t|, 2}=\left\{\zeta_{2}|| \zeta_{2}|<| t \mid\right\}$ of the punctures $p_{1}$ and $p_{2}$ (respectively) and then, for $|t|<\epsilon$, we form the (complex) identification space $\mathcal{R}_{t}=\left(\mathcal{R}_{0}-\left(U_{t, 1} \cup U_{t, 2}\right)\right) /\left\langle\zeta_{1} \zeta_{2}=t\right\rangle$. The space $\mathcal{R}_{t}$ is a Riemann surface of genus one more than the genus of $\mathcal{R}_{0}$ but with two fewer punctures, and is said to be the result of the conformal plumbing of $\mathcal{R}_{0}$. A similar operation forms, from $\mathcal{R}_{0}^{\prime}$ and $\mathcal{R}_{0}^{\prime \prime}$, a new surface $\mathcal{R}_{t}^{\prime}$ with genus the sum of the genera of $\mathcal{R}_{0}^{\prime}$ and $\mathcal{R}_{0}^{\prime \prime}$ and with two fewer punctures than the total of those on $\mathcal{R}_{0}^{\prime}$ and $\mathcal{R}_{0}^{\prime \prime}$.

We now describe the minimal area metric on $\mathcal{R}_{t}$ in terms of the minimal area metric on $\mathcal{R}_{0}$; the case of the minimal area metric on $\mathcal{R}_{t}^{\prime}$ is analogous. So let $\rho_{0}$ be the minimal area metric hypothesized throughout this paper; if $\rho_{0}$ is smooth and complete near the punctures $p_{1}$ and $p_{2}$, then we have seen that $\rho_{0}$ is flat in some neighborhood of $p_{i}$ and foliated there by geodesics of length $2 \pi$.

We perform our plumbing using the canonical domain $\mathcal{U}_{i}$ around the puncture $p_{i}$, defined by the minimal area metric (see our discussion at the beginning of $\S 5.1)$. The domain $\mathcal{U}_{i}$ is foliated by geodesics homotopic to $p_{i}$, and is conformally equivalent to the punctured disk $\left\{\left|\zeta_{i}\right|<1\right\}$. We take $\mathcal{U}_{|t|, i}$ to be the domain parametrized by the subdisk $\mathcal{U}_{|t|, i}=\left\{\left|\zeta_{i}\right|<|t|\right\}$ (note a small change in notation from $\S 5.1)$.

Next we form, as above, the identification space $\mathcal{R}_{t}=\mathcal{R}_{0}-\left(\mathcal{U}_{|t|, 1} \cup \mathcal{U}_{|t|, 2}\right) /\left\langle\zeta_{1} \zeta_{2}=t\right\rangle$. Now, the surface $\mathcal{R}_{t}$ admits the alternative description as the smooth surface obtained by gluing together the ends of $\mathcal{R}_{|t|^{\text {cut }}}=\mathcal{R}_{0}-\left(\mathcal{U}_{|t|^{1 / 2}, 1} \cup \mathcal{U}_{|t|^{1 / 2}, 2}\right)$ corresponding to $p_{1}$ and $p_{2}$, the gluing taking place along the curves $\left|\zeta_{1}\right|=|t|^{1 / 2}$ and $\left|\zeta_{2}\right|=|t|^{1 / 2}$ and the identification being made so that $\zeta_{1} \zeta_{2}=t$ along the seam $\Gamma=\left\{\left|\zeta_{1}\right|=|t|^{1 / 2}=\left|\zeta_{2}\right|\right\}$. We observe that the curves $\left|\zeta_{1}\right|=|t|^{1 / 2}$ and $\left|\zeta_{2}\right|=|t|^{1 / 2}$ are geodesics in the metric $\rho_{0}$ which is flat near the seam, so that the restriction of the metric $\rho_{0}$ to $\mathcal{R}_{0}-\left(\overline{\mathcal{U}_{|t|^{1 / 2}, 1}} \cup \overline{\mathcal{U}_{|t|^{1 / 2}, 2}}\right)$ extends to a smooth metric $\rho_{0, t}$ on $\mathcal{R}_{t}$ 
which is flat near the seam.

Corollary 5.7. For $|t|<e^{-2 \pi}$, the plumbed surface $\mathcal{R}_{t}$ admits a minimal area metric $\rho_{t}$ and $\rho_{t}=\rho_{0, t}$.

Proof. We first observe that $\rho_{0, t}$ is a candidate metric on $\mathcal{R}_{t}$. This is clear once we consider the homotopically non-trivial curves on $\mathcal{R}_{t}$. If $\gamma$ is such a curve then either $\gamma$ meets the seam $\Gamma$ or it does not. If $\gamma$ does not meet $\Gamma$, then we may take $\gamma$ to be a curve on $\mathcal{R}_{0}$. Moreover, this curve $\gamma \in \mathcal{R}_{0}$ must be non-trivial, and hence have length at least $2 \pi$ : if $\gamma$ is trivial in $\mathcal{R}_{0}$ it must bound a disk $D \subset \mathcal{R}_{0}$; but then $D$ is contained in $\mathcal{R}_{|t|}^{\text {cut }}$ since $D$ cannot contain the punctures and $\gamma=\partial D$ does not meet the seam $\Gamma$. In that case actually $D \subset \mathcal{R}_{t}$ and $\gamma$ would be trivial in $\mathcal{R}_{t}$, in contradiction with the initial assumption that $\gamma$ was nontrivial. If $\gamma$ meets the seam $\Gamma$ and one of the arcs $\left\{\left|\zeta_{i}\right|=1\right\}$, then $\gamma$ must either cross the union of the domains $\left\{\left|\zeta_{1}\right| \geq|t|^{1 / 2}\right\} \cup\left\{\left|\zeta_{2}\right| \geq|t|^{1 / 2}\right\}$ or one of those subdomains twice, in either case acquiring a $\rho_{0, t}$ length of at least $2 \pi$, as long as $|t|<e^{-2 \pi}$. If $\gamma$ meets the seam $\Gamma$ but is properly contained in a neighborhood of the seam of size $\pi$, then $\gamma$ must be homotopic to the seam, and one sees easily that such a curve has a length of at least the length of $\Gamma$, or $2 \pi$.

Next we see that $\rho_{0, t}$ has least area among admissible metrices on $\mathcal{R}_{t}$. From Theorem 5.1 (Amputation), we see that $\rho_{0, t}$ is the minimal area metric on $\mathcal{R}_{|t|}^{\text {cut }}$. Then, if there were an admissible metric $\rho_{t}$ on $\mathcal{R}_{t}$ with lower area than $\rho_{0}$, then the metric $\rho_{t}$ would restrict to be a metric on $\mathcal{R}_{|t|}^{\text {cut }}$ of area lower than $\rho_{0, t}$. Yet the metric $\rho_{t}$ would still be admissible for the minimal area problem on $\mathcal{R}_{t}^{\text {cut }}$, since any homotopically non-trivial curve $\gamma$ on $\mathcal{R}_{t}^{\text {cut }}$ is a nontrivial curve on $\mathcal{R}_{t}$. To see this, either use Van Kampen's theorem, or more concretely, suppose $\gamma$ is trivial in $\mathcal{R}_{t}$, then it bounds a disk $D \in R_{t}$; since $\gamma$ does not meet $\Gamma$ on $\mathcal{R}_{t}^{\text {cut }}$ (this is easily arranged by a small deformation) either $\Gamma$ is fully contained in $D$ or it is completely disjoint from $D$. However, $\Gamma$ cannot be contained in $D$, since $\Gamma$ is a nontrivial curve, therefore $D$ is a proper disk in $\mathcal{R}_{t}^{\text {cut }}$, and this implies that 
$\gamma$ is trivial in $\mathcal{R}_{t}^{\text {cut }}$ in contradiction with the assumption that it was a nontrivial curve. Thus the metric $\rho_{t}$ would be both admissible and of area lower than $\rho_{0, t}$ on $\mathcal{R}_{t}^{\text {cut }}$. This contradiction shows that $\rho_{0, t}$ is of minimal area on $\mathcal{R}_{t}$, concluding the argument.

Remark. This corollary provides for the existence of solutions to the minimal area problem for surfaces of higher genus using minimal area metrics of lower genus through the process of plumbing. The resulting Riemann surfaces correspond to a deleted neighborhood of the compactification divisor of $\overline{\mathcal{M}}_{g, n}$. For example, it was shown in $\left[\mathrm{Zw}_{w}\right]$ that the minimal area problem was solvable for all punctured spheres $\mathcal{R}=S^{2}-\left\{p_{1}, \ldots, p_{n}\right\}$, the solution being given as the norm of a holomorphic Jenkins-Strebel quadratic differential on $\mathcal{R}$. This corollary then provides for the existence of solutions to the minimal area problem of surfaces obtained by the plumbing of a number of punctured spheres. Of course, in this particular case one sees that these solutions are also given by Jenkins-Strebel quadratic differentials.

\section{Minimal Area Metrics not Arising from Quadratic Differentials}

In this section we will give an example of a minimal area metric solving the generalized minimal area problem. The unusual aspect of this minimal area metric is that it does not arise from a quadratic differential. This will be manifest since we will get negative curvature singularities corresponding to an excess angle of $\pi / 2$. In quadratic differentials the excess (or defect) angle must be an integer multiple of $\pi$ (an $n$-th order zero corresponding to an excess angle of $n \pi$ ). Moreover the pattern of foliations by geodesics of lengths $2 \pi$ is also novel. For a minimal area metric arising from a Jenkins-Strebel quadratic differentials one can always take the horizontal trajectories of the differential to define the foliating geodesics. Then the surface is completely foliated by geodesics that do not intersect (geodesics ovelap along the critical graph of the quadratic differential, this graph, of course, has zero measure). In the example to be discussed it is not possible to cover the surface with saturating geodesics that do not intersect. This is probably the crucial 
feature of all the metrics solving the generalized minimal area problem which do not arise from quadratic differentials.

Let us now describe the surface and its metric. The surface will turn out to be a genus five surface $\mathcal{R}$ with no punctures. It will be constructed by gluing together two identical tori $\mathcal{T}$ and $\mathcal{T}^{\prime}$ each with four boundaries. Each torus with boundaries is given by a $2 \pi$ by $2 \pi$ square region in the $z$ plane, with the natural flat metric $\rho_{0}=1$ on it, and with opposite edges identified to give a torus. We cut four square holes on each torus, each square of perimeter $2 \pi$ and symmetrically centered, as shown in Fig. 5(a). These are the four boundaries. The tori $\mathcal{T}$ and $\mathcal{T}^{\prime}$ are joined by four short flat tubes $\mathcal{C}_{i}$ (not shown in the figure) attached to the boundary components. Each tube is a cylinder of circumference $2 \pi$ and height $\pi / 2$. Note that at the corners of the square holes the metric $\rho_{0}$ has curvature singularities with an excess angle of $\pi / 2$.

Let us verify that the metric is admissible. All nontrivial curves homotopic to curves lying completely in $\mathcal{T}$ or $\mathcal{T}^{\prime}$ are good because they are longer than or have lengths equal to some homotopic curve lying completely in $\mathcal{T}$ (or $\mathcal{T}^{\prime}$ ) and such curves are manifestly good. Curves homotopic to a core curve in one of the short tubes also belong to this class. We also need to consider simple closed Jordan curves going from $\mathcal{T}$ to $\mathcal{T}^{\prime}$. These curves must travel along the tubes an even number of times. Since the tubes have height $\pi / 2$ the only case we need to consider is that of curves going across two times, thus acquiring at least length $\pi$. Suppose they go up and down the same tube. If we delete the two segments going up and down the tube we obtain two open curves. Each curve is longer than or equal to an open curve of the same homotopy type that does not enter the tube in question again. Consider those open curves, which are defined on $\mathcal{R}$-(tube). At least one of them should be a nontrivial open curve since otherwise the original closed curve is either trivial or homotopic to a core curve in the tube. A nontrivial open curve, however, must be larger than $3 \pi / 2$ (check the figure). Thus we exceed the necessary length. The only curves left to consider are those that meet two different tubes. Since the distance between the different boundary components in 
$\mathcal{T}$ and $\mathcal{T}^{\prime}$ is $\pi / 2$ the curve must gain an extra $\pi$ of length, and therefore must be good.

Before showing the metric is of minimal area let us describe the pattern of foliations. These are indicated in the figure and are of three types. The first type is foliations lying completely in $\mathcal{T}$ or in $\mathcal{T}^{\prime}$. There are four bands of foliations and they cross, covering $\mathcal{T}$ (or $\mathcal{T}^{\prime}$ ) once in some regions and twice in others (Fig. 5(b)). The second type of foliations are those extending both in $\mathcal{T}$ and $\mathcal{T}^{\prime}$. They are indicated in Fig. 5(c) and the geodesics go from one boundary component in $\mathcal{T}$ to another, then up the tube to $\mathcal{T}^{\prime}$, then to another boundary component in $\mathcal{T}^{\prime}$ and then down the tube to $\mathcal{T}$. Such curves have length $2 \pi$. They extend over the regions of $\mathcal{T}$ and $\mathcal{T}^{\prime}$ where the first type of foliations gave a single covering. They also cover the tubes once. There are eight bands of this type. The third type of foliations are those whose geodesics are homotopic to the core curves in the tubes, there are four such bands, one in each tube (not shown in the figure). The three types of foliations put together give a double covering of the complete surface $\mathcal{R}$.

Let us now prove that the metric is of minimal area. Beurling's criterion (see [Ah]) applied to our problem says that a metric $\rho_{0}$ solves the generalized minimal area problem if it is admissible and there is a family of nontrivial closed curves $\Gamma_{0}$ such that $l_{\rho_{0}}(\gamma)=2 \pi$ for all $\gamma \in \Gamma_{0}$, and for any real valued $h$ in $\mathcal{R}$ such that

$$
\int_{\gamma} h|d z| \geq 0,
$$

for all $\gamma \in \Gamma_{0}$, we have that

$$
\iint_{\mathcal{R}} h \rho_{0} d x d y \geq 0 .
$$

Let us show our metric on $\mathcal{R}$ satisfies this criterion. The family $\Gamma_{0}$ consists of the three types of foliations discussed above. Each foliation covers a annular region $\mathcal{R}_{i}$ of the surface, isometric to a flat strip of length $2 \pi$ with edges identified. Put 
rectangular coordinates $x, y$ on each strip, and let the geodesics correspond to the constant $x$ lines. It then follows from (6.1) that

$$
\int d y \int d x h \geq 0, \quad \text { and so } \quad \iint_{\mathcal{R}_{i}} h \rho_{0} d x d y \geq 0
$$

since $\rho_{0}=1$. Adding over all foliations we obtain

$$
\sum_{i} \iint_{\mathcal{R}_{i}} h \rho_{0} d x d y=2 \iint_{\mathcal{R}} h \rho_{0} d x d y \geq 0,
$$

since all foliations together give a double covering of the surface. This concludes our proof that the metric $\rho_{0}$ in $\mathcal{R}$ is of minimal area.

Remark. There is an interesting one parameter deformation of the above metric [Ro]. We can vary the heights of the short tubes continuously but in doing so we must keep the heights of diametrically opposite tubes equal, and the sum of heights of neighboring tubes equal to $\pi$. All these metrics are of minimal area. The endpoint of this deformation is a configuration where two of the tubes collapse and the other two tubes become of height $\pi$ each. This metric does not arise from a quadratic differential nor we can cut the tubes and still have a minimal area metric. (Had the tubes been longer than $2 \pi$ we could have cut them and obtain a minimal area metric.)

Acknowledgements: The authors benefitted from interesting conversations with R. Forman, T. Maskawa, K. Ranganathan and M. Rocek.

\section{REFERENCES}

[Ah] Ahlfors, L. V.: Conformal Invariants, topics in geometric function theory. New York: McGraw Hill, 1973. 
[Ber] Bers, L.: Spaces of Degenerating Riemann Surfaces.: Annals of Math Studies, No.79, Princeton University Press, Princeton, NJ, 1974, 43-55.

[Beu] Beurling, A.: Collected Works of A. Beurling. Eds. L. Carleson et.al. Boston: Birkhauser, 1989.

[EL] Eells, J., Lemaire, L.: Deformations of Metrics and Associated Harmonic Maps, Patodi Memorial Vol. Geometry and Analysis (Tata Inst., 1981), $33-45$.

[EM] Earle, C.J., Marden, A.: Geometric Complex Coordinates for Teichmuller Space. To appear.

[Ga] Gardiner, F.: Teichmuller theory and quadratic differentials. New York: John Wiley 1987.

[Ha] Harer, J.: The cohomology of the moduli space of curves, in Theory of Moduli: Lectures given at C.I.M.E. Springer Lecture Notes in Mathematics, 1337. Springer Verlag, Berlin, 1988.

[H] Hartman, P: Ordinary Differential Equations. Wiley, Baltimore, 1973.

[Hi] Hicks, N.: Notes of Differential Geometry, Van Nostrand, Princeton, 1965.

[Je] Jenkins, J. A.: On the existence of certain general extremal metrics. Ann. Math. 66440 (1957).

$[K K S]$ T. Kugo, H. Kunitomo and K. Suehiro, Nonpolynomial Closed String Field Theory. Phys. Lett. 226B 48 (1989)

$[K S]$ T. Kugo and K. Suehiro.: Nonpolynomial closed string field theory: action and gauge invariance. Nucl. Phys. B337 434 (1990)

[Mas] Masur, H.: The Extension of the Weil-Petersson metric to the boundary of Teichmuller Space. Duke Math. J43 (1979), 623-635.

[Ra] Ranganathan, K.: A criterion for flatness in minimal area metrics that define string diagrams. Comm. Math. Phys., to appear.

[Ro] M. Rocek, private communication. 
[SaZw] M. Saadi and B. Zwiebach.: Closed string field theory from polyhedra. Ann. Phys. 192213 (1989).

$[S o Z w]$ H. Sonoda and B. Zwiebach.: Closed string field theory loops with symmetric factorizable quadratic differentials. Nucl. Phys. B331 592 (1990).

[Sp] Spivak, M.: A Comprehensive Introduction to Differential Geometry.: Publish or Perish Press, Berkeley, 1979.

[St] Strebel, K.: Quadratic Differentials. New York: Springer-Verlag, 1984.

$[W]$ Wolf, M.: Infinite Energy Harmonic Maps and Degeneration of Hyperbolic Surfaces in Moduli Space. J. Diff. Geom. 33, 487 (1991),

[Zw1] Zwiebach, B.: How covariant closed string theory solves a minimal area problem.: Comm. Math. Phys. 136, 83 (1991); Phys. Lett. B241, 343 (1990).

[Zw2] Zwiebach, B.: Quantum closed strings from minimal area.: Mod. Phys. Lett. A5, 2753 (1990).

[Zw3] Zwiebach, B.: Minimal area problems for quantum open strings. Comm. Math. Phys. 141, 577 (1991).

[Zw4] Zwiebach, B.: Recursion relations in closed string field theory, Proceedings of the "Strings 90" Superstring Workshop. Eds. R. Arnowitt, .et al. (World Scientific, 1991) pp. 266-275.

[Zw5] Zwiebach, B.: Quantum closed string field theory: Action and BRST transformations. In preparation. 


\section{Figure Captions}

Figure 1 . The segments $A_{0}$ and $A_{0}^{\prime}$ make up the $\rho_{0}$-geodesic $\gamma_{0}$. The segments $A_{\epsilon}$ and $A^{\prime}{ }_{\epsilon}$ make up the $\widehat{\rho}_{\epsilon}$-geodesic. The segments $A_{0}$ and $A_{\epsilon}$ are homotopic $\operatorname{rel}\left\{p_{1}, p_{2}\right\}$. Interpolating between $A_{0}$ and $A_{\epsilon}$ we show some $\widehat{\rho}_{t}$-geodesics $A_{t}$, where $\widehat{\rho}_{t}=\rho_{0}+t h$.

Figure 2. We show a collection of geodesics $\gamma(\cdot, t)$ with $t \in[0, \kappa]$. The geodesics are seen to converge because the variation field $V(s)=\frac{\partial}{\partial t} \gamma(s, t)$ is not constant: $\frac{\partial}{\partial s}\|V(s)\| \neq 0($ at $q)$. The domains $R_{+}$and $R_{-}$where the deformation of the metric is supported, are indicated.

Figure 3 . We show a curve $\gamma$ that penetrates a ring domain $F_{i}$, isometric to a flat cylinder of circumference $2 \pi$, a distance $\delta$. To the right we open up the flat annulus bounded by $\mathcal{C}_{0}$ and $\mathcal{C}_{\delta}$.

Figure 4. A minimal area metric complete and smooth near a puncture determines a canonical domain $\mathcal{U}$ around the puncture. The boundary of $\mathcal{U}$ is $\mathcal{C}_{0}$. This canonical domain is isometric to a flat semiinfinite cylinder. The curve $\mathcal{C}_{\delta}$, a distance $\delta$ away from $\mathcal{C}_{0}$, divides the surface $\mathcal{R}$ into $\mathcal{R}_{\delta}$ and $\mathcal{U}_{\delta}$. Also indicated is the stub $S_{\delta}$. The amputation theorem relates the minimal area metric on the amputated surface $\mathcal{R}_{\delta}$ to the original minimal area metric on $\mathcal{R}$.

Figure 5. A genus five surface $\mathcal{R}$ with a minimal area metric that does not arise from a quadratic differential is built by joining together two tori, each with four boundaries, using four short tubes. (a) A torus $\mathcal{T}$ (or $\mathcal{T}^{\prime}$ ): the edges of the square domain are identified and the four square holes are the boundaries. (b) The pattern of foliations that are completely contained in $\mathcal{T}$ (or $\mathcal{T}^{\prime}$ ). (c) A partial view of the foliations that extend both in $\mathcal{T}$ and $\mathcal{T}^{\prime}$. 